\title{
3 Schwierigkeiten und Triumph einer Überhöhung
}

\section{Zur Erzählkunst im Rolandslied}

Résumé : L’article tente de décrire avec toute la précision possible les problèmes narratifs qui se présentaient lors de la dernière grande transformation de la Chanson de Roland, c'est-à-dire lors de l'addition de la partie Blancandrin et de la partie Baligant, de la refonte du procès de Ganelon et de l'élaboration de la nouvelle fin sophistiquée - changements tous dus, semble-til, à un seul et même poète de génie.

Wie lang oder kurz man sich die Vorgeschichte des erhaltenen Rolandsliedes ${ }^{1}$ auch vorstellen mag - heute gibt es wohl keinen Forscher, der dem Lied jede Vorgeschichte abspricht, es sich gleichsam dem Haupt des Dichters entsprungen denkt wie Athene dem Haupt des Zeus. Und da die Entwicklung des Stoffes doch von dem historischen Kern des Liedes, dem Untergang der fränkischen Nachhut, ihren Ausgang genommen haben dürfte, drängt sich der Schluss auf, dass es einmal ein Lied noch ohne Blancandrin-Szene, ohne Baligant-Teil, mit einem nur rudimentären Prozess gegen Ganelon ${ }^{2}$ und ohne die Schlussverse um Vivien, Bire und Imphe gab.

Andererseits sollte nach den Forschungen der letzten etwa siebzig Jahre unbestritten sein, dass jener Literat - nenne man ihn Dichter, Bearbeiter oder wie immer -, der das erhaltene Lied ,verantwortet‘, das heißt, in essentiell der auf uns gekommenen Form aus der Hand gab, ein hochgebildeter Kleriker war und ohne diese Bildung seinem Werk nicht jene Dichte und Tiefe hätte geben

1 Die gängige Bezeichnung ,Oxforder Roland“ ist insofern missverständlich, als nicht der Text der Oxforder Handschrift (O), sondern ein fast immer aus $\mathrm{O}$, doch unter ständigem Vergleich der sonstigen Überlieferung erstellter Text gemeint ist, wie ihn etwa Cesare Segre bietet in den recte gedruckten (nicht in den kursiv gedruckten, in $\mathrm{O}$ fehlenden) Laissen seiner von mir im Folgenden stets zitierten Edition La Chanson de Roland, nouvelle édition revue, traduite de l'italien par Madeleine Tyssens, 2 Bde., Genf 1989, die substantiell nicht verschieden ist von der italienischen Erstauflage La Chanson de Roland, edizione critica a cura di Cesare Segre, Mailand 1971. (Bei Segres partienweise doppelter Verszählung gebe ich stets die nicht-eingeklammerten Zahlen, die $\mathrm{O}$ ohne Laissenumstellungen folgen.)

2 Mir ist unklar, ob und wie lange ein Rolandslied ohne Verrat und Strafe, also noch ganz ohne Ganelon, lebensfähig gewesen wäre; deshalb gehe ich nicht bis zu einer solchen Stufe zurück.

Anmerkung: Erstmals veröffentlicht in: Romanistisches Jahrbuch 59 (2008), 128-156.

Ә Open Access. (c) 2019 Gustav Adolf Beckmann, publiziert von De Gruyter. (c))BY-NC-ND Dieses Werk ist lizenziert unter der Creative Commons Attribution-NonCommercial-NoDerivatives 4.0 Lizenz.

https://doi.org/10.1515/9783110615692-003 
können, die es hinaushebt nicht nur über die anderen chansons de geste, sondern zugleich über die ganze Epoche früher romanischsprachiger Dichtung. ${ }^{3}$ Ohne dass wir völlig sicher sein können, dass Blancandrin-Szene, Baligant-Teil, die jetzige Form von Ganelons Prozess und erst recht die Schlussverse von ein und demselben Dichter stammen - über diese Problematik sind ja viele hundert Seiten geschrieben worden -, berechtigt uns doch die bloße Tatsache, dass das Lied so erhalten ist, bis zum Beweis des Gegenteils dazu, es als Einheit zu würdigen; die Beweislast für das Gegenteil läge bei den Chorizonten. Aber selbst wenn die genannten Teile nicht von demselben Dichter stammen sollten, ist es lohnend, sie systematisch auf ihre narrative Leistung innerhalb des Gesamtwerkes und zugleich auf die Probleme zu befragen, die ihr Einbau in die Handlungsfolge mit sich brachte.

Das soll im Folgenden versucht werden. Dabei scheint mir gerade die letztgenannte Fragestellung, die nach den ,Einbauproblemen', in der Forschung bisher nicht ausgeschöpft. Hier möchte ich unter anderem aufzeigen, wie die Technik, den Rezipienten naheliegende Fehlperspektiven zu verbauen - oder, mit einem anderen Bilde gesagt, wie das Vermeiden narrativer Klippen - einen nicht unwesentlichen Teil der Erzählkunst ausmacht, die hinter dem erhaltenen Rolandslied steht.

Im Pseudo-Turpin finden wir bekanntlich keine Blancandrin-Episode. Die Hinführung zur Roncevaux-Handlung ist einfacher als im Rolandslied: Karl hat ganz Spanien außer Saragossa erobert; bevor er nun gegen die Stadt vorgeht, schickt er Ganelon als Boten mit der Aufforderung zur Taufe oder Tributzahlung. Eine solche Botschaft wird nahegelegt wohl schon durch ein rudimentäres ius gentium, sicher durch Karls christliche Grundhaltung und seinen Willen zur Vermeidung unnötiger eigener Verluste. Da hier die Franken vom Denken des Marsirus (und seines Bruders Beliguandus) gar nichts wissen, kann Ganelons Leben wirklich in Gefahr sein. Marsirus braucht nur eine Verteidigung der Stadt

3 Freilich macht dieses Lied es der heutigen Leserschaft oft nicht leicht, ex ungue leonem zu erkennen. Ich denke insbesondere daran, wie vermeintliche ad-hoc-Einfälle, ,Bildungslücken` oder Bizarrerien unvermutet einen tiefen Sinn offenbaren können. Exemplarisch für einen nur scheinbaren $a d$-hoc-Einfall ist etwa die Mitteilung, im Ebro seien mehr Gegner ertrunken als von den fränkischen Verfolgern getötet wurden (v. 2477), für eine scheinbare Bildungslücke die (Erz-) Engeltrias Gabriel-Cherubin (statt Rafael!)-Michael (v. $2389 \mathrm{ff}$.), für eine scheinbare Bizarrerie die Erwähnung von Vergil und Homer (v. 2616, nur in O, doch ins Original gehörig, da $O$ sonst nie inhaltliche Zusätze enthält). Vgl. zu diesen Stellen meinen Artikel „Le vers, le verset et le contexte : Encore le Roland d'Oxford et la Bible“, Romania 122 (2004), S. 532-542. 
bis zum letzten Blutstropfen zu planen, und es würde ihn nichts kosten, als Trotz- und Warngebärde gegen Karl dessen Boten abzuschlachten. Insgesamt ist diese Fassung des Stoffes ebenso einfach wie logisch, und schon weil sich in der Forschung der letzten Jahrzehnte die Tendenzen zur Spätdatierung beim Rolandslied stärker als beim Pseudo-Turpin ausgewirkt haben, wird sich erneut die alte Frage melden, ob die einfache Fassung nicht auch die ältere ist. Doch ist die Prioritätsfrage für das Folgende im Prinzip irrelevant; es kommt nur darauf an, dass die beschriebene Handlungsstruktur auf Grund ihrer Einfachheit jedem Bearbeiter des Themas mindestens für einen Augenblick in den Sinn gekommen sein muss, auch wenn er schließlich eine komplexere Lösung vorzog, wie dies der Rolanddichter tut. Was gewinnt er eigentlich durch die Blancandrin-Episode? Zumindest zweierlei.

Erstens kennen wir hier Marsilies wahres Denken. Dadurch wird für uns die ganze erste Ratsszene der Franken gleichsam doppelbödig, was gleich für die ersten ,Diskussionsbeiträge“ darin entscheidend wichtig ist. Roland widerspricht als Wortführer weiteren Krieges seinem kaiserlichen Onkel recht aufbrausend (ne l'otriët mie [...], li vint cuntredire, v. $194 \mathrm{ff}$.), während Ganelon (v. $217 \mathrm{ff}$.) trotz kaum verschleierter Beleidigungen gegen Roland ein humaneres Ziel, nämlich das Wohl des ganzen Heeres, im Auge zu haben scheint - aber wir wissen: Objektiv ist Roland im Recht, nicht Ganelon.

Zweitens ermöglicht Blancandrins Botengang eine der psychologisch eindrucksvollsten Szenenfolgen der mittelalterlichen französischen Literatur: das Weggespräch zwischen ihm und Ganelon (v. 366-406) und Ganelons Auftreten vor Marsilie (v. 407-660). Ganelon hatte zwar schon in der Ratsszene durch die Diskrepanz zwischen seinem Friedensplädoyer und seiner anschließenden Panik das Gesicht verloren und glaubte es nur zurückgewinnen zu können, indem er in Gegenwart der ganzen Versammlung zunächst Roland seine Feindschaft für den Fall seiner Rückkehr aus Saragossa ankündigte (v. 289-291), dann seine Fehdeansage auf die zwölf Pairs ausdehnte (v. 322-326). Doch die Tatsache, dass sich weder der Kaiser noch die Ratsmitglieder über diese Ankündigungen entsetzten, erweist, dass alle zunächst nur an die übliche Privatfehde dachten, die den Lehnsherrn der befehdeten Person oder Personen nicht in Mitleidenschaft ziehen und selbstverständlich erst nach dem Ende des gemeinsamen Kriegszugs in Kraft treten durfte. Ganelon zieht zu seinem Botengang voller Hass aus, aber noch ist es ein dunkler Hass in potentia, nicht in actu. Da tritt der Versucher $\mathrm{zu}$ ihm in einer Geste von, man möchte sagen, auffälliger Unauffälligkeit: Blancandrin bleibt hinter den anderen sarazenischen Boten einige Schritte zurück und gesellt sich zu Ganelon (v. 368). Wie einst bei der Ursünde der Menschheit Eva und Adam nicht spontan sündigten, sondern des Versuchers bedurften, so auch hier. Und wie dort der Versucher zunächst ein unver- 
fänglich wirkendes breiteres Thema ansprach - „Ihr dürft nicht von den Früchten der Bäume essen?“ - so auch hier (v. 370-374, 377-380): „Ein wundersamer Mann ist Karl; er hat so viele Länder erobert! Weshalb reicht ihm dieser Ruhm nicht endlich?" Doch Ganelon ist nicht willens, den Kaiser selbst zu verleumden (v. 375 f.), Blancandrin muss etwas genauer werden (v. 378-380): „Die Kriegstreibereien seiner Großen müssen doch letztlich den Franken selbst zum Schaden gereichen!?“ Jetzt offenbart die einengende Antwort der oder des zu Verführenden die entscheidende Schwachstelle, wie einst: „Gott hat uns nur einen einzigen Baum verboten,“ so jetzt (v. 380 f.): „Nur Roland ist der eigentliche Kriegstreiber." Von nun an kann der Versucher auf dieser Schwachstelle insistieren und damit Ganelon eine zunehmend reale Perspektive zu Rolands Vernichtung eröffnen. Schließlich geloben noch auf dem Wege Ganelon und Blancandrin einander, gemeinsam auf Rolands Tod hinzuarbeiten. Da dies mitten im Krieg geschieht, ist der Charakter einer Privatfehde überschritten: Ganelon gelobt einen Verrat, hat ihn aber noch nicht ausgeführt. Denn noch ist die Umsetzung dieser Absicht für ihn alles andere als einfach. Blancandrin führt ihn zwar, an der Hand haltend, bei Marsilie ein als noble barun und mult riches hom (v. 415, 421 f.), Gesten, die ihn als Boten aufwerten und zugleich diskret andeuten sollen, dass sich hier eine unerwartete Entente anbahnt. Aber um den bisher ja zutiefst kampfunwilligen Marsilie zur Aufbietung aller Kräfte gegen Roland umzustimmen, muss Ganelon ihm panischen Hass gegen Roland einpflanzen. Dafür bleiben ihm nur wenige Augenblicke; deshalb muss er zunächst, seine Vollmacht überschreitend, Marsilie demütigen und ihm Roland vorstellen im Schreckbild einer Teilung Spaniens (v. 432) mit Roland als „mult orguillos [...] parçuner“ (v. 473f.). ${ }^{4}$ Das Manöver kostet ihn fast sein Leben; doch für eine schlechte Sache bringt er jetzt - psychologisch durchaus glaubhaft in einer ihm dämonisch zuwachsenden Kraft den Mut und sogar die äußere Contenance auf (v. 467: „Noble baron ad ci!"*), die ihm jüngst für eine gute Sache fehlten. Seine Rechnung geht auf: Man kann Marsilie knapp zur Mäßigung bringen, er zieht sich mit seinen engsten Getreuen zur Beratung in den Garten zurück. Dort klärt ihn Blancandrin so auf, dass sogar Ganelons Provokation vergeben und vergessen ist um der größeren Perspektive willen: Der Franke (v. 507) „de nostre prod m’ad plevie sa feid.“ Bei Ganelon erkundigt sich nun auch Marsilie zunächst eindringlich nach dem ihm bekannten Feind, eben Karl, mit der dreimaligen hyperbolischen Formulierung, Karl müsse doch nach all

4 Die Passage hat zu divergierenden Interpretationen Anlass gegeben, vgl. etwa Gerard J. Brault, The Song of Roland, Bd. I, Introduction and Commentary, University Park PA 1978, S. 144-155, 399-401 (mit Lit.). Die obige Interpretation scheint sich mir durch Einfachheit zu empfehlen. 
seinen Feldzügen inzwischen wohl zweihundert oder mehr Jahre alt sein (v. 524, 539, 552). Heute würde man, wenn man ebenfalls hyperbolisch sein wollte, etwa sagen: ,Karl führt doch nun schon seit endlosen Zeiten einen Krieg nach dem andern.' Doch wieder hütet sich Ganelon vor jedem bösen Wort über Karl (v. 529-536) und engt stattdessen das Gespräch zielstrebig ein auf Roland (v. 544550, 557-562), dann auf die Möglichkeit, ihn zu töten (v. 567-579, 582-600). Schließlich beschwört er ohne Zögern den Verrat auf die Reliquien seines Schwertes (v. 608): „La traïson jurat, si s'est forsfait.“5 Denn selbst wenn er jetzt in einem Anflug von Furcht oder Reue in der zweiten Ratsszene Roland nicht zum Führer der Nachhut vorschlüge, hätte er Verrat schon dadurch begangen, dass er Marsilies gesamte Heeresmacht auf die Nachhut lenkte.

Man stelle sich nun statt dieser durch zunehmende Dämonie aufgeladenen Ereignisfolge vor, wie plump ein allein eintreffender Ganelon wäre, der sich kurz nach seiner Ankunft selbst als Verräter anbiedern müsste - und man wird für die Blancandrin-Episode dankbar sein. Die Handlungsfolge im PseudoTurpin zeigt gesunden Menschenverstand, die Blancandrin-Episode Genie. ${ }^{6}$

5 Als in der ersten Hälfte des zwanzigsten Jahrhunderts der Historismus etwa eines Friedrich Meinecke das europäische Denken beherrschte in Richtung auf ein ,Alles verstehen ist alles verzeihen', mag es auch Ansätze zu einem ,humanist misreading (so der griffige Terminus von Roger Pensom, Literary Techniques in the Chanson de Roland, Genf 1982, S. 127) von Ganelons Charakter gegeben haben. Dagegen hat dann die zweite Jahrhunderthälfte zunehmend opponiert, am stärksten mit dem Tenor ,ein bloßer Verräter‘, „,completely evil“ ohne einen „redeeming trait whatsoever“, Brault (wie Anm. 4), passim, speziell S. 100-103. Ich halte es jedoch für entscheidend wichtig, dass sich der Dichter weder bei Ganelon noch im umgekehrten Sinne etwa bei Bramimonde oder Roland über seinem emphatisch-eindeutigen Endurteil davon dispensiert, vorher sein ganzes psychologisches Talent aufzuwenden auf die Nachzeichnung des Weges zu diesem Endpunkt. Unterscheidet man Weg und Endpunkt nicht schon prinzipiell, so übersieht man eine der wichtigsten Dimensionen dichterischer Größe des Oxforder Rolands; denn dann hebt sich dessen Ganelon kaum noch ab von dem Ganelon der späteren Rolandfassungen und anderer Epen, der in der Tat bei jeder Gelegenheit ein bloßer Verräter ist, würdiger Spross eines Clans, der schon Julius Cäsar umgebracht hat. Wie weit der Dichter seinen Ganelon von einem solchen Erbbiologismus entfernt sah, zeigt ein kleines, aber eindrucksvolles Wort: Ganelons (durch die Saisnes-Epik bestätigte) Gewissheit, dass sein leiblicher Sohn „ert prozdoem“ (v. 314). Zu Bramimondes Entwicklung siehe den Haupttext weiter unten. Zu Roland sei hier nur bemerkt, dass Gott selbst noch dem todwunden Helden seinen letzten ,weltlichen`Wunsch, die Zerstörung seines Schwertes (v. 2297 ff.), abschlägt und dass Roland erst nach langer Reflexion über seine Eroberungen und nach dem dritten und letzten Versuch der Zerstörung sich der Reliquien im Schwert erinnert, die es ,hochheilig“ (seintisme, v. 2344) machen und, so man denn an sie glaubt, eine Zerstörung des Schwertes in merkwürdigem Licht erscheinen lassen müssten.

6 Angesichts dieser Errungenschaften der Blancandrin-Szene sollte es nicht schwerfallen, im Gegenzug zuzugeben, dass bei ihrem Einbau in das ältere Handlungsgefüge kleine Unebenheiten geblieben sind. Doch sind sie das? Wenn von Marsilies zehn Boten (v. 63-68) höchstens 
Doch sozusagen auf der Rückseite dieses erzählerischen Triumphes sind für den Erzähler zwei gefährliche Klippen, nämlich für sein Publikum zwei naheliegende narrative Fehlperspektiven, entstanden.

Erste Frage: Warum schickt nicht Karl einfach Blancandrin mit einem Ultimatum an Marsilie zurück, ohne einen seiner Männer zu gefährden? Eine gerade in ihrer Banalität gefährliche Frage; denn Roncevaux läuft Gefahr, nicht stattzufinden. Bédier hat zwar aus der Realität des Ersten Kreuzzuges einen Fall beigebracht, wo Gesandte und Gegengesandte hin- und hergesandt werden: Von Antiochia aus stellen die Kreuzfahrer den noch fernen Kalifen von Kairo vor die Wahl, entweder ihr Feind oder aber ihr Bundesgenosse gegen die Türken $\mathrm{zu}$ werden, die ihm kurz vorher Jerusalem abgenommen haben. ${ }^{7}$ Doch da sind beide Parteien zunächst bereit, gleichsam auf Augenhöhe zu verhandeln. Vor Saragossa ist das nicht so: Marsilie steht sichtlich mit dem Rücken gegen die Wand. Dass hier eine Gegengesandtschaft keineswegs zu erwarten war, lässt der Rolanddichter selbst durchblicken, wenn der heimkehrende Blancandrin meldet (v. 418-422): „Vostre message fesime[s] a Charlun ; / Ambes ses mains en levat cuntre munt. / Loat sun Deu, ne fist altre respuns. / Ci vos enveiet un sun noble barun / Ki est de France, si est mult riches hom : / Par lui orrez si avrez pais $\mathrm{u}$ nun." Man hat also eher mit einer Antwort Karls an Blancandrin als mit dem Erscheinen Ganelons gerechnet. Anders ausgedrückt: Der Dichter hat diese Erzählklippe durchaus wahrgenommen.

Wie schafft er es dann, dass sie den Rezipienten nicht zum Bewusstsein kommt? Er schafft es, indem er uns nachhaltig daran erinnert, dass im Mittelalter der kluge Bote auch eine Art Späher sein konnte. Wer bis zum feindlichen König vorgelassen wurde, konnte en passant manches beobachten zur Stärke und Moral des Heeres, zur Persönlichkeit und jetzigen Befindlichkeit des Herrschers, vor allem zur Möglichkeit und zum Stand der Verteidigung gegen eine Belagerung. Einen eigenen klugen Boten zu schicken, konnte sich also auszahlen als Erkenntnisgewinn. In diese Richtung lenkt Karl gleich zu Anfang der ersten Ratsszene das Denken seiner Berater durch den Satz (v. 191): „Mais jo ne sai quels en est sis curages.“ Es wird also darum gehen, mehr von Marsilies wahrem Denken zu erfahren, und dieses Mehr wird man nicht aus Blancandrin herausholen können. Deshalb schlägt sich gerade Naimes, Inbegriff des klugen

ein einziger später als Kämpfer wiedererscheint (Est<r>amarin, KV4 -iz v. $64 \sim$ Estramariz v. 941, 1304), so kann sich der Dichter die Boten überwiegend als würdige Senioren jenseits des Kampfalters vorgestellt haben. Die (h)ostages wiederum erscheinen noch in v. 572, 646, 679, also bis ins ,Nachfeld“ der Blancandrin-Szene; ,vergessen“ sind sie erst im Schlussteil des Liedes.

7 Joseph Bédier, Les légendes épiques, 3. Aufl., Bd. III, Paris 1929, S. 404. 
Königsfreundes, als erster für den Botengang vor (v. 246); doch Karl möchte seinen einzigen Intimus in der Nähe behalten. Es folgt Roland, den aber Olivier sofort wegen seiner Unbeherrschtheit als ungeeignet bezeichnet - und Karl schließt durch ein Machtwort gleich alle zwölf Pairs aus; hier sind keine Heißsporne gefragt. Jetzt schlägt sich mit klarerer Begründung Turpin vor (v. 269 f.): „E jo irai al Sarazin espa[n], / Si·n vois vedeir alques de sun semblant.“ Karl will in der Tat Näheres über Marsilies semblant erfahren, aber nicht über dessen Götzendienst, er braucht die Augen eines Diplomaten. Vielleicht erraten nun schon viele, wen er im Sinn hat, wagen aber aus hierarchischer Scheu nicht, des Kaisers Schwager zu benennen; nur einer, der in der Hierarchie genau so hoch, dem Kaiser mindestens genau so nahe steht, wagt es (v. 277): „Ço ert Guenes, mis parastre.“ Damit wird es von einem Augenblick zum andern ungefährlich, im Chor zu bestätigen (v. 278f.): „Car il le poet ben faire! / Se lui lessez, n'i trametrez plus saive.“ Der Bote muss also vor allem saive sein. Und noch einmal wird später vorausgesetzt, dass der eigene Bote, so er denn saive ist, unschätzbare Informationen bringen kann - oder vielmehr könnte: bei Ganelons Lügenbericht vom Meerestod des Algalife und seiner Leute (v. 681-691).

Hat das Publikum des Liedes somit am Nutzen eines eigenen Boten keine Zweifel mehr, so liegt doch dicht neben der ersten narrativen Klippe eine zweite. Unterstellt man nämlich einen intellektuell und emotional leidlich normalen Marsilie, so ist im Rolandslied anders als im Pseudo-Turpin Ganelons Mission schlechthin gefahrlos. Zwar wissen die Franken nicht, ob Marsilie es mit seiner Bitte, gerade in Aachen getauft $\mathrm{zu}$ werden, ehrlich meint oder nicht. Aber eines wissen sie genau: Marsilie will sie zum Rückzug bewegen. Ist er aufrichtig, so kann Ganelon seine diplomatische Mission ehrenvoll zum Erfolg führen. Ist er es nicht, so würde ein ,normaler' Marsilie auch die kurze Anwesenheit des Boten noch heuchelnd durchstehen, schließlich geht es für ihn um alles oder nichts. Dieser elementaren Logik könnte sich auch der intelligente Ganelon nicht verschließen; er könnte nicht mehr glauben, von Roland in den Tod geschickt zu werden, und würde sich hüten, nur wegen eines für ihn blamablen Wortstreits seine Existenz durch Verrat aufs Spiel zu setzen. Abermals droht Roncevaux auszufallen.

Um auch diese Klippe zu umschiffen, das heißt, um den Rezipienten diesen Denkweg früh genug $\mathrm{zu}$ verlegen, muss Marsilie als blutrünstig und unbeherrscht bis zur Selbstschädigung dastehen, und zwar von Beginn der ersten Ratsszene an, damit schon während der Szene Ganelon die Sendung nach Saragossa als Todesurteil empfinden kann (v. 311): „Hom qui la vait, repairer ne s'en poet.“ Wie schafft der Dichter das? Genial einfach: Gleich im ersten Diskussionsbeitrag zur Ratsszene erfahren wir, dass Marsilie schon einmal so gehandelt hat (v. 201-209). „Li reis Marsilie i fist mult que traïtre. / De ses paien«s vos en 
en veiat quinze, / Cha[s]cuns portout une branche d'olive; / Nuncerent vos cez paroles meïsme. / A voz Franceis un cunseill en presistes; / Loërent vos alques de legerie. / Dous de voz cuntes al paien tramesistes; / L'un fut Basan e li altres Basilies; / Les chef en prist es puis desuz Haltilie.“ Marsilies spätere Zornesanfälle (v. 438 ff., 485 ff.) wirken dann wie eine Bestätigung von v. 201-209. Doch schon mit diesen Versen selbst sollte die alte Hoffnung mancher Epenforscher, dass schon vor dem Rolandslied ,von Basan und Basilie‘ gesungen wurde, dahin sein; zu eindeutig ist der Text Satz für Satz in die Situation hineingeschrieben. Mehr noch: Diesem Grafenpaar gibt der Dichter alliterierende Namen, wie er sie auch sonst liebt, so bei Gerin und Gerer, Ive und seinem Freund Ivórie (gerade jenen vier unter den zwölf Pairs, von denen wir weder den Rang noch das Lehen kennen, die also wohl zur Auffüllung der Zwölfzahl paarweise hinzuerfunden sind), bei Machiner und seinem Onkel und Mitboten Maheu, Clarifan und seinem Bruder und Mitboten Clariën, Esturganz und seinem Genossen Estramariz samt einem weiteren Genossen Escremiz, Malcud und seinem Sohn Malquidant. Dazu kommt eine offenbar ad hoc erfundene Hinrichtungsstätte, unidentifizierbar und mit redendem Namen: „es puis desuz Haltílie“ (so O, v. 209; „souz Montoïe“ V7, „soz Aute-vile“ C) oder „as puis de Haltoïe“ (so O, v. 491, „Montoïe“ V 7, „Aute Hoï̈“ C), also bei ,Hohenhausen“ oder ,Hohenecho“. ${ }^{8}$ Kurzum, nirgends finden wir in dieser Szene die Spur eines Archaismus, stattdessen durchgängig die Handschrift und eine klare Erzählmotivation des Dichters.

$\mathrm{Zu}$ dieser Erkenntnis liefert unfreiwillig Paul Aebischer eine Gegenprobe. Er möchte die Episode um Basan und Basilie schon einer vor dem Rolandslied liegenden, heute verlorenen Entrée d'Espagne zuschreiben. ${ }^{9}$ Wie begründet er

8 An der zweiten Stelle verlangen beide Stemmazweige Haltoïe gegen Segres Emendation Halt[il]ie, an der ersten steht $\mathrm{O}$ gegen V7, C hat vielleicht eine Mischform (Rhythmus aus Haltoïe, -l-aus Haltílie). Will man nun nicht annehmen, V7 oder seine Vorlage habe in erstaunlicher Sorgfalt die weiter hinten stehende, als richtig empfundene Lesung zurückblätternd vorn hineinkorrigiert, so gehört Haltö̈e mit Stengel, Hilka und Roncaglia an beiden Stellen in den Text. Auch inhaltlich kann es bestechen durch eine ähnliche akustische Sensibilität, wie die berühmten Hornszenen sie bezeugen; Haltílie kann dann einfach durch Basílie am Ende des vorhergehenden Verses beeinflusst sein. Freilich könnte man Haltílie auch aus dem (schlecht datierbaren) Typ Altillo der spanischen Kleintopographie herleiten und deshalb für primär halten. Fernzuhalten ist hingegen aus chronologischen wie geographischen Gründen das italienische Attilie des Otinel.

9 Paul Aebischer, „Deux récits épiques antérieurs au Roland d'Oxford: l'Entrée d'Espagne primitive et le Girard de Viane primitif“, Études de lettres [Genf], III 1 (1968), S. 4-35 (wiederabgedruckt bei P. Ae., Des Annales carolingiennes à Doon de Mayence. Nouveau recueil d'études sur l'épique française médiévale, Genf 1975, S. 131-158, hier S. 141-143), und ders., Textes norrois et littérature française du Moyen Âge, II, La première branche de la Karlamagnús-Saga, Genf 1972, hier S. 84-91, das oben folgende Zitat S. 85. 
dann Marsilies Verhalten? „Ne sachant plus à quelle idole se vouer, Marsile envoie des ambassadeurs à Charlemagne, lui disant qu'il était disposé à se faire chrétien, à condition qu'on lui laisse son royaume. Charles, heureux de cette solution, charge Basin et son frère Basilius de notifier à Marsile son complet accord; mais on ne sait trop pourquoi - peut-être par suite de cette logique diplomatique arabe qui parfois nous échappe - le roi de Saragosse, au comble de la colère, fait saisir et mettre à mort les deux envoyés impériaux. “ Ganz recht, auf der Ebene des Erzählten nous échappe jede Erklärung für Marsilies Verhalten - weil sie eben auf der Ebene des Erzählers, des Rolanddichters als Erfinders der Blancandrin-Episode, liegt.

Kommen wir von Blancandrin zu Baligant! Der Baligant-Teil trägt in die alte Fabel das neue Weltgefühl der Kreuzzugszeit hinein, insbesondere deren mächtig erweiterten geographischen Horizont, einen großenteils durchkämpften, also ,erlebten“ Horizont, innerhalb dessen die bisherige Beschränkung des Liedhorizontes auf Spanien samt einem nordafrikanischen Anhang schlechthin provinziell wirken musste. ${ }^{10}$ Der Dichter konnte Roncevaux nicht glaubhaft in den Orient verlegen, so holte er umgekehrt - gewissermaßen das Ei des Kolumbus den Orient nach Spanien hinein. Und da sich dieses neue ,universale“ Weltgefühl viel eher an der Gestalt eines idealen universalen Monarchen ${ }^{11}$ als an der Person des Führers einer Nachhut festmachen ließ, überhöht der Baligant-Teil das Rolandsdrama zum Karlsdrama, ja zu einem Ringen zwischen Christentum und Heidentum schlechthin.

Das Baligant-,Problem“ ist oft als Dichotomie gesehen worden: Stammt das erhaltene Rolandslied von einem oder von zwei Dichtern? Wenn diese seit $1877^{12}$ geübte Fragestellung bis heute zu keinerlei Konsens geführt hat, so wohl, weil sie eo ipso eine Annäherung zwischen beiden Positionen erschwert.

10 Kennzeichnend für den Bewusstseinsumschwung vom elften zum zwölften Jahrhundert ist beispielsweise, dass nach der Eroberung von Jerusalem Papst Paschalis II. Anfang des zwölften Jahrhunderts Spaniens Rittern und Klerikern mehrfach die Teilnahme an Orientexpeditionen verbieten musste, damit Spanien nicht von Kämpfern entblößt werde (Jaffé-Löwenfeld 5839 f., 5863, letzteres noch um die Jahrhundertmitte verbatim aufgenommen in die Historia Compostellana [ed. Emma Falque Rey, Turnhout, 1988], Buch 1, Kap. 39). Also galt selbst bei Spaniern - und a fortiori, so muss man schließen, bei Franzosen - der im Orient zu gewinnende Ruhm weit mehr als der in Spanien zu gewinnende.

11 Schon durch das Bewusstsein der Teilnehmer des Ersten Kreuzzuges geistert ja die Idee eines Karolus Magnus resuscitatus, dazu etwa Ekkehard von Aura (vor 1107), MGH, Scriptores, Bd. 6, S. 215.

12 Vgl. Bédiér (wie Anm. 7), S. 393. Einen rezenten gedrängten Überblick über die jüngere Forschungsgeschichte und die Problematik des Baligant-Teils findet man bei Mary Jane 
Linguistische (grammatische oder lexikalische) Unterschiede zwischen beiden Teilen des erhaltenen Rolandsliedes darf man in Abrede stellen; ${ }^{13}$ vielmehr beeindruckt gerade, dass ein so unübertrefflich guter Kenner des Textes wie Segre passim von ,der' Sprache ,des' Rolandsliedes redet. Bei Annahme zweier Autoren sollte man deshalb zumindest mit einem ungewöhnlich kongenialengen Verhältnis rechnen, in dem der ,Schüler ' den ,Lehrer` vor allem überragt, weil er auf dessen Schultern steht.

Aber auch umgekehrt: Optiert man im Prinzip für die Autoreinheit, so heißt das noch keineswegs, dass das Werk nach vorgegebenen normativen Kriterien ,aus einem Guss‘ sein müsste. Der Dichter könnte nach Jahren, sogar Jahrzehnten auf Grund seiner neuen kreuzzugsdominierten Vision sein Werk überarbeitet und ausgeweitet haben; schon das könnte z. B. einen etwas pompöseren Stil des Baligant-Teils als Altersstil erklären. ${ }^{14}$ Aber selbst wenn er beide Teile bald nacheinander schrieb, verlangte der Übergang von ihm eine teilweise neue poetische Technik.

Im Roland-Teil waren ihm zumindest Grundzüge der Handlung vorgegeben, und er wird auch an mancher sprachlichen Formulierung, an manchem Einzel-

Schenck, „The Baligant Episode“, in: Approaches to Teaching the „Song of Roland“, edd. William W. Kibler und Leslie Zarker Morgan, New York, 2006, S. 213-219.

13 Hier genügt es, sich an die beiden exemplarischen Diskussionen zu erinnern: Suchier glaubte, der Baligant-Teil trenne /ẽ/ und /ã/, das übrige Lied vermische sie; aber Bédier (wie Anm. 7), S. 399 Anm. 1, zeigte, dass die Proportionen in beiden Teilen nur wenig verschieden sind. (Vgl. auch Bédiers spätere, etwas subtilere Darstellung in La Chanson de Roland commentée, Paris 1927, S. 278-280.) Und die Vokabular- und Formelunterschiede, die Jules Horrent, La Chanson de Roland dans les littératures française et espagnole au moyen âge, Paris 1951, festzustellen glaubte, wurden von seinem Lehrer Maurice Delbouille, Sur la genèse de la Chanson de Roland: essai critique, Brüssel 1954, S. 46-57, in minutiöser und überzeugender Argumentation durch geeignete Parallelen als zufällig erklärt. (Anschließend, S. 57-61, bringt Delbouille auch Argumente für eine Übereinstimmung der Laissentechnik. Doch siehe dazu genauer den Haupttext weiter unten mit Anm. 18!) Die Gefahr bei zu weiter Öffnung des linguistischen ,Mikroskops“ besteht eben darin, dass man unter die Signifikanzschwelle, in den Bereich des plausiblerweise Zufälligen, gerät. Zur Probe teile man einen beliebigen Text von der Länge des Rolandsliedes in der Mitte; man wird immer, mindestens mit Hilfe des Computers, sprachliche ,Unterschiede‘ zwischen beiden Hälften finden können.

14 Ich denke hier speziell an die richtige Charakterisierung des Baligant-Stils als „plus orné, plus fleuri“ bei Horrent (wie Anm. 13), S. 253-254, und die Erklärung dieses Sachverhalts aus der Verfassereinheit in Delbouilles Erwiderung (wie Anm.13), S. 44-45. (Auch die übrigen Argumente von Delbouilles Kapitel „De l'authenticité de Baligant“, op. cit., S. 32-44, bleiben lesenswert.) - Darf ich hier vergleichshalber zu einem albtraumhaften Denkexperiment einladen? Im Jahr 2700 seien Faust I und Faust II unbeschädigt zusammen überliefert und im Wortsinn gut verständlich, aber alle Zeugnisse von Goethes Autorschaft verloren. Welcher Stilforscher wird wagen, ein solches Werk als verantwortete Einheit zu betrachten? 
motiv seines Vorgängers gehangen haben. Im Baligant-Teil hingegen musste er die Handlung neu entwerfen, wobei er sich anscheinend locker an militärische Ereignisse im Nordspanien der Jahre 1110-1118 anlehnte, speziell an den überraschenden und zunächst sehr bedrohlichen Vorbruch der auf Afrika gestützten Almoraviden nach Saragossa. ${ }^{15}$

Doch vor allem: Ihm musste klar sein, dass er, wenn er seine Rezipienten nicht ermüden wollte, die grandiosen Dimensionen der Baligantschlacht weit gedrängter als die Schlacht von Roncevaux würde darstellen müssen. Ich weiß nicht, ob die Beobachtung, so naheliegend sie ist, je gemacht worden ist: Im erhaltenen Rolandslied mit seinen 4002 Versen liegt der Goldene Schnitt a maiore (d. h. mit dem längeren Teil als erstem) in v. 2474; das ist der letzte Vers, der vom Untergang der Flüchtigen am verlängerten Tag berichtet. Dann zieht der Laissenschlussvers 2475 die Bilanz dieser Episode: „Franceis escrïent: - Mar [veï]stes Rollant!“ Mit v. 2476 beginnt die Laisse, mit der Delbouille ${ }^{16}$ den Baligant-Teil einsetzen lässt: Karl beschließt an Ort und Stelle jene Übernachtung, während deren er den Baligant-Traum hat. Wie anscheinend das ganze Mittelalter ${ }^{17}$ kannte zweifellos auch der Dichter des erhaltenen Liedes den Begriff des Goldenen Schnittes nicht, beweist aber einen ähnlichen Sinn für Proportionen: Ob man nun den Beginn der Baligant-Handlung hier oder etwas später ansetzt auch nach der ,Überhöhung‘ wird der Roland-Teil des Liedes jedenfalls vom Nach-Roland-Teil nicht erdückt. Und wer so die Proportionen zu wahren versteht, ist sich zwangsläufig auch der Notwendigkeit größerer Gedrängtheit im Baligant-Teil bewusst. Deshalb finden wir jetzt keine laisses similaires mehr, auch die sonstigen verbal-repetitiven Verknüpfungen aufeinander folgender Laissen, die ja ebenfalls Raum kosten, nehmen gegenüber dem Roncevaux-Teil

15 So wohl richtig André de Mandach, Naissance et développement de la Chanson de geste en Europe, Bd. 6, Chanson de Roland, Genf, 1993, S. $184 \mathrm{ff}$. - Falls man der im Pseudo-Turpin sichtbar werdenden Tradition auch die zeitliche Priorität zubilligt, wie das ja bei den heutigen Datierungen ohne Weiteres möglich ist, hätte der Autor des erhaltenen Rolandsliedes zudem, um nicht ganz als Erfinder ,wilder Märe‘ dazustehen, den Beliguandus, der auch im PseudoTurpin aus Persien Babylonien kommt und im Gegensatz zu Marsirus Roncevaux überlebt, seinen Zwecken dienstbar gemacht mit einer leichten ,Biblisierung und damit Orientalisierung des Namens (Bel- > Bal- nach den biblischen Babylonierkönigen Baladan und Balthassar [so die Vulgata für ,Belsazar'], vielleicht auch in vagerer Assoziation nach Gestalten wie Balac und Balaam [so die Vulgata für ,Bileam']), was zugleich bei der Einführung Baligants (v. 2614) die einprägsame Alliteration En Babilonie Baligant ermöglichte.

16 Wie Anm. 13, S. 33.

17 Günter Binding im Lexikon des Mittelalters, 9 Bde., Studienausgabe, Stuttgart 1999, s.v. Proportionen. 
im Verhältnis 34 : 54 ab. ${ }^{18}$ Vor allem aber müssen jetzt die Zweikämpfe, die geradezu das Hauptingrediens des Roncevaux-Teils waren, sparsamer und zugleich gezielter eingesetzt werden: So illustrieren vom Beginn der Schlacht an einzelne knapp gehaltene Zweikämpfe (v. 3352-3368, 3463-3470) zunächst Sieg, dann tragischen Tod von dreien der vier Führer der fränkischen Jungmannschaft; von der Mitte der Schlacht an führt dann eine ebenfalls eng strukturierte Folge von drei Zweikämpfen (v. 3421-3450: Naimes tötet Baligants Sohn, wird beinahe von Baligants Bruder getötet, aber von Karl gerettet) über die momentane Gefahr eines feindlichen Durchbruchs (vv. 3529, 3533) zu dem einzigen breiter ausgeführten Zweikampf, dem schlachtentscheidenden zwischen Karl und Baligant (v. 3564-3620).

Stattdessen tritt jetzt, obgleich ständig kunstvoll verwoben mit einheimischen Motiven, ein struktureller Einfluss der Antike klarer hervor als vorher, ein generelles Wissen darum, wie Höhepunkt und Schlussteil einer großen epischen Handlung auszusehen haben. ${ }^{19}$

So kann das Duell zwischen Karl und dem Herrn der Heidenheit zwar durch eine Fassung des Sachsenkriegs angeregt sein; denn ein legendäres Duell zwischen Karl und Widukind als Höhepunkt und Schluss von Karls Sachsenkrieg findet sich schon um 974 und um 1002 in den beiden ältesten Mathildenviten, ${ }^{20}$ dann um 1200 in Bodels Saisnes, vermutlich also auch in untergegangenen Zwischenstufen. Doch zugleich dürfte, poetisch legitimierend, das Herrscherduell als gattungsspezifisches Finale antiker Großepik, etwa der Äneis oder der Thebais - und warum nicht auch aus der Alexanderliteratur Alexanders Duell mit Porus? - im Hintergrund stehen.

$18 \mathrm{Zu}$ beidem, sachlich interessant, wenn auch die Relevanz des Fehlens der laisses similaires meines Erachtens überbetonend, Joseph J. Duggan, The Song of Roland: formulaic style and poetic craft, Berkeley CA 1973, S. 98-100. Bemerkenswert übrigens, dass auch Duggan, obwohl er schließlich knapp für Autorentrennung optiert, den Formelschatz beider Teile als großenteils gemeinsam erweist: Auf 526 Eigenformeln des Roncevaux-Teils und nur 81 Eigenformeln des Baligant-Teils kommen 300 gemeinsame Formeln, so dass Duggan (op. cit., S. 84, vgl. auch 97) schließt, der Baligant-Teil habe kein eigenes Formelrepertoire.

19 Insgesamt frappante Parallelen zwischen der Aeneis und auch den vorhergehenden Teilen des Rolandsliedes brachte zwar schon Bédier (wie Anm.13), S. 316 f., bei; er bemerkt aber: „Mais, chose très digne de remarque! jamais le rapprochement n'est assez précis pour qu'on puisse affirmer que notre auteur a directement, consciemment imité.“ Eine beeindruckende Reihe von Parallelen auch bei André Burger, Turold, poète de la fidélité, Genf 1977, S. 73-81.

20 MGH, Scriptores, Bd. 10, S. 576, und Bd. 4, S. 284 f. Dieser Tatbestand wird manchmal selbst in Untersuchungen, die ausdrücklich der Vorgeschichte der Saisnes-Epik gewidmet sind, übersehen, z. B. bei Paul Aebischer, „L'élément historique dans les chansons de geste ayant la guerre de Saxe pour thème“, in: P. Ae., Des Annales carolingiennes (wie Anm. 9), S. 223-239. 
In dieselbe Richtung scheinen Einzelszenen zu weisen. Baligants Bruder Canabeus ist ein ,Hunde-bell-o' (can[is] + abai[er],aboyer ${ }^{`}+-u s$ ) vielleicht nur in deformierender Namenskomik; denn macht man eine angenommene Metathesis rückgängig, so lässt sich ${ }^{*}$ Cabaneus, wie schon Jenkins vermutete, ${ }^{21}$ schwer trennen von dem Capaneus (Handschriften-Variante auch Cabaneus) der Thebais, wobei das tertium comparationis darin liegt, dass beide noch buchstäblich in der Sekunde, in der sie zum sieghaften Schlag bzw. Sprung ansetzen, durch göttliches Eingreifen gefällt werden.

In der Episode um Jangleu, den ,Kritikaster` (v. 3507-3519, afrz. jangler ,quengeln, laut oder häufig tadeln' + -aeus), bliebe dessen Beiname l'ultremarin merkwürdig unspezifisch, wenn er (in der Perspektive des Dichters) bedeutete ,mit den mehr als eineinhalb Millionen Vasallen Baligants soeben aus dem Orient herübergekommen' und nicht vielmehr (in Baligants Perspektive), einst aus dem Okkzident in den Orient herübergekommen'; im selben Sinne auffällig sind Jangleus distanzierende Worte an Baligant „Morz estes“ statt Morz somes und vor allem ,vostre deu“ statt nostre deu. Es liegt doch wohl die geringfügige Variation eines Erzählmusters vor, das bis in die griechische Geschichte zurückreicht (Herodot 7.101-105: Xerxes befragt den Griechen Demaratos; lateinisch Curtius 3.2.10-19: Darius fragt den Athener Charidemos) und das in der Schilderung der Schlacht vor Antiochia (1098) bei Raimond d'Aguilers (cap. 17) und Fulcher von Chartres (1.22.4-8), also vor 1106, auch auf Kerbogha übertragen erscheint, der einen aus dem christlich besetzten Antiochia entflohenen Türken befragt. Jeweils steht der orientalische Potentat vor einer großen Schlacht (oder einer ganzen Kampagne), die er auf Grund seiner materiellen Überlegenheit zu gewinnen hofft, und will sich seine Aussichten noch eben von einem bei ihm weilenden Kenner seiner okzidentalischen Gegner bestätigen lassen; doch der betont rückhaltlos deren Kampfgeist und sagt dem Potentaten ein völliges Scheitern voraus, das dann auch eintritt.

À propos Curtius: Ich vermute, dass auch die unmittelbar vor der Schlacht in Baligants Gegenwart zelebrierenden zehn ,kanaanäischen' (Canelius) Götzenpriester mit ihren religiösen Gesängen (v. 3269 ff.) direkt oder (eher) indirekt inspiriert sind durch Curtius 3.3.9, wo in ähnlicher Situation (der persischen Heerschau) und ebenso prominenter Position „magi [die Angehörigen der Priesterkaste des Reiches] patrium carmen canebant“ - abgesehen im Rolandslied natürlich von dem Namen ,Kanaanäer', den sie als Nachkommen der prototypischen Götzendiener des Alten Testaments tragen. ${ }^{22}$

21 La Chanson de Roland, Oxford Version, edition, notes, and glossary by Thomas Atkinson Jenkins, 2. Aufl., Boston 1929, ad v. 3312.

22 Gerade angesichts der zunehmenden Spätdatierungen des Rolandsliedes scheint mir ein minutiöser Vergleich mit der gesamten auf Latein zugänglichen Alexander-Literatur ein Desi- 
Geradezu das Markenzeichen antik-epischen Einflusses sind schließlich die beiden Truppenkataloge (v. 3026-3095, 3217-3264), wie sie ja seit Homer in kaum einem antiken Epos, in der Regel zu Beginn des Endkampfes, fehlen. Freilich überbietet der Dichter seine Vorbilder strukturell, indem er auf christlicher Seite zwischen dem A und $\mathrm{O}$ des reichstragenden Frankenvolkes die anderen Landsmannschaften in ungefährer Kreuzesform aufzählt ${ }^{23}$ und auf heidnischer Seite manche der realgeographischen Namen umbiegt zu negativ-wertenden (speziell Farb-) Adjektiven, so dass Baligants Scharen von der Bosheit und dem fahlen Schein der Hölle durchglüht erscheinen. ${ }^{24}$

derat der Forschung zu sein. Vgl. auch einen so merkwürdigen Einzelfall wie den weiter unten behandelten des Justin de Valferree!

23 Zugleich übernimmt er aus der realen Schlacht vor Antiochia 1098 den ethnisch definierten eschele-Begriff (vgl. Bédier, wie Anm. 13, S. 53f.) samt der charakteristischen Schluss- (nicht Spitzen- oder Mittel-) Position des Oberbefehlshabers (dort also Bohemunds Position nach dem übereinstimmenden Zeugnis von Gesta, Raimund von Aguilers, Anselm von Ribemont, Petrus Tudebodus, Tudebodus imitatus, Robert von Reims, Albert von Aachen, Ordericus Vitalis, Wilhelm von Tyrus und Chanson d'Antioche) in der quantitativ stärksten eschele (so explizit Albert von Aachen und Wilhelm von Tyrus). Baudri de Dol erklärt sogar ausdrücklich: Aciei sextae praesedit Boamundus, ut omnibus praevideret atque singulorum in necessitatibus adesset. Denn da die größte Gefahr in einer solchen Schlacht darin besteht, dass der Gegner irgendwo einen Durchbruch erzielt und aus der Flanke heraus Truppenteile in panische Flucht treibt, muss der Oberbefehlshaber zunächst für den Notfall mit einer starken Eingreiftruppe im Hintergrund bleiben; nur so kann Karl dann ja auch den feindlichen Durchbruch (v. 3528 f., 3533) noch eindämmen. Entsprechend nimmt auch Baligant bei cil d'Ociant (v. 3286), also der letzten eschele des zentralen, rückwärtig platzierten (s. folgende Anm.!) Heeresdrittels (v. 3246), eine entsprechende Schlussposition ein; das befähigt auch ihn, seine eigens gebildete Eingreiftruppe (v. 3283-3285) gegen Ende der Schlacht einzusetzen (v. 3517-3519). Zugleich hat diese strategisch motivierte Schlussposition beider Oberbefehlshaber den dichterisch erwünschten Nebeneffekt, dass beide physisch erst spät, doch dann gegeneinander und damit schlachtentscheidend eingreifen können.

24 Ich akzeptiere alle diesbezüglichen Bemerkungen in den Aufsätzen Alfred Noyer-Weidners, wie sie am leichtesten zugänglich sind in seinem Umgang mit Texten, Bd. I, hrg. von Klaus W. Hempfer, Stuttgart 1986, S. 1-148, speziell 1-64. Doch glaube ich im Gegensatz zu NoyerWeidner gerade nicht, dass damit die konkurrierenden realgeographischen Bezüge hinfällig sind; obwohl z. B. die Avěrs auch ,Gottwidrige‘ und die Pers ,(Höllisch-) Fahlblaue‘ sind, stehen dahinter doch die Avaren, die Perser usw. Der Dichter juxtaponiert nicht hin- und herspringend Reales und Symbolisches zu einer anderweitig unbelegten Mischästhetik (deren Jeu sogar recht fazil wäre, denn wieso kommen die ,Widrigen“ gerade an sechzehnter, die ,Fahlblauen“ an dreizehnter Stelle...?). Vielmehr folgt er - was hier freilich aus Platzgründen nicht im Einzelnen belegt werden kann - einem einfachen realgeographischen Schema: Die ersten zehn escheles, von der Baligantschen Westperipherie stammend (Osteuropa bis Nordostafrika), bilden den linken Vorderflügel seiner Schlachtordnung; die dritten zehn escheles, von der (zwangsläufig in Vagheit oder Phantasie ausklingenden) Baligantschen Ostperipherie, bilden den rechten Vorderflügel; die mittleren zehn escheles, aus Baligants Schwerpunktzone (etwa 
Insgesamt gibt es also zwischen beiden Teilen des Rolandsliedes keine sprachlichen Unterschiede, hingegen merkliche erzähltechnische. Da ich bei ihnen jedoch mit einer bemerkenswerten Fähigkeit ,des‘ Dichters zur erzähltechnischen Anpassung an seine neue Vision rechne, halte ich Autoreinheit für das Wahrscheinlichere. Andere mögen umgekehrt urteilen - doch in beiden Perspektiven bleibt für uns Heutige die poetologische Schwelle zwischen Roncevaux- und Baligant-Teil.

Für jene große Mehrheit der mittelalterlichen Rezipienten, die sich noch keineswegs zur Anerkennung einer ,Legitimität der Fiktion' durchgerungen hatte, war es sogar eine viel stärkere Schwelle: aus einem geliebten, leidlich ,geglaubten' Terrain hinaus in eine Welt unverbürgten narrativen Neulands. Und wenn das Fehlen des Baligant-Teils im Pseudo-Turpin originär sein kann, so scheint es mir in der Karlamagnús-Saga und in späteren Textzeugen wie dem niederländischen Roelantslied oder der Roland-Hs. L so gut wie sicher auf bewusster Elimination, das heißt, auf Ablehnung zu beruhen..$^{25}$ Selbst dass im 13. und 14. Jh. die Rolandslied-Überlieferung hinter der Pseudo-Turpin-Überlieferung quantitativ extrem zurückbleibt, könnte außer auf dem Latein-Bonus und der Autorfiktion des Pseudo-Turpin auf einem verbreiteten Misstrauen gegenüber der Baligant-Handlung beruhen.

Auch hier ist also mit der großen neuen Vision und sozusagen in ihrem Rücken eine banale Gefahr entstanden: dass viele Rezipienten den Baligant-Teil global als ,wilde Märe‘ verwerfen. Sie kann auch dem Dichter nicht verborgen geblieben sein. Deshalb bereitet er im Roncevaux-Teil den Übergang, von langer Hand' vor, freilich so dezent, dass es heutigen Rezipienten meist entgeht. Machen wir in diesem Sinne einmal Bilanz!

Schon in v. 89 f. schickt Marsilie Karl „dis blanches mules [...] / Que li tramist li reis de Suatilie“ - wo Metrum und Assonanz die Lesungen Sua-ti-li-e und Su-a-tí-lie zulassen. ${ }^{26}$ Maultiere können zwar überall gezüchtet werden, wo es

Persien bis Bulgarien), sind hinter beiden aufgestellt, und bei ihrer zwanzigsten und damit letzten Schar steht Baligant selbst (vgl. Anm. 23). Doch durch leichte Namendeformationen in Richtung auf die Adjektiv- und speziell Farbsymbolik macht der Dichter zusätzlich ,Tiefe im Antlitz der Welt' sichtbar. Diese Vertiefungsästhetik (durch negative Interpretation realer Namen), nicht eine Juxtapositionsästhetik (des willkürlichen Nebeneinanders realer und symbolischer Namen) ist durch die mittelalterliche Bibelinterpretation gedeckt. Übrigens scheint mir die ,fahlblaue' und verwandte höllische Farbsymbolik nicht erst mittelalterlich zu sein, sondern aus Apoc. 9. 14-17 zu stammen, wo zum Klang der sechsten Posaune (also spät, aber noch vor Anbruch des Endgerichts) das über den Euphrat (!) andringende Heer durch loricas igneas, et hyacinthinas, et sulphureas gekennzeichnet ist.

25 Siehe jetzt G.A. Beckmann, Die Karlamagnús-Saga I und ihre altfranzösische Vorlage, Tübingen 2008, S. 197-200.

26 Açil rei de Cecilie, Sizilien` V 4 ist sichtlich lectio facilior. 
Pferde und Esel gibt, doch hier geht es um besonders edle, weiße Tiere, offenbar ein kostbar-charakteristisches Geschenk ihres Heimatlandes. Am ältesten und berühmtesten ist die Maultierzucht Kleinasiens. ${ }^{27}$ Denkt der Autor an sie, so liegt der Einschiffungshafen der Tiere am ehesten an der Südküste der heutigen Türkei - und dort gibt es nur einen Hafen ersten Ranges, das antike Attáleia (so die griech. Betonung im Nom. und Akk., Act. Apost. 14.25: eis Attáleian), heute Antalya, doch lat. Attalia und bei den nichtgriechischen Christen seit dem frühen 12. Jh. durchweg Satalía, Satellía u. ä. ${ }^{28}$ Antalya war im 8. oder 9. sowie im späten 11. oder frühen 12. Jh. mehrfach muslimisch besetzt, sonst bis 1207 doch lange ohne das Umland - in byzantinischer Hand. ${ }^{29}$ Obwohl das $-u$ - in 0 unerklärt bleibt, ist an der Identifikation kaum zu zweifeln. Marsilie steht also in freundschaftlicher Verbindung mit muslimischen (Teil-) Herrschern des Orients.

Auch Marsilies Gold ist „or d’Arabe“ (v. 185, 652), sein Faltstuhl bespannt mit einem „paile Alexandrin“ (v. 408).

Dem Margariz de Sibilie „tramist li amiralz de Primes“ sein Schwert (v. 967). Die Forschung nimmt Primes als Toponym, ohne eine diskussionswürdige Hypothese anzubieten. Im Rolandslied ist amiralz der höchste muslimische Titel - kann da ein amiralz an einem relativ kleinen, unidentifizierbaren Ort sitzen? Geht es nicht einfach wie v. 589, 1924, 2845 um das Adverb primes ,vorher, zuerst‘, hier also de primes etwa ,einst‘? Dann wurde dem Margariz sein Schwert einst einfach von ,dem' amiral $z$, herübergesandt ${ }^{*}{ }^{30}$

27 Nordkleinasien (Ilias 2.852), Mysien (Ilias 24.276, Anakreon), Galatien (Luxustiere, Plutarch, De cupidit. divit. 2), Phrygien (Hieronymus und danach Rhabanus Maurus in Ez. 27.14 [Migne, Patrologia Latina, Bd. 25, S. 253 f., Bd. 110, S.775]), Lydien (Äsop). Ausführliche Nachweise in Pauly-Wissowas Realencyclopädie, Bd. 6 (Halbd. 11), Sp. 659, s. v. Esel.

28 Mit einem $S$-, das abstrahiert ist entweder aus (ei)s Attáleian (vgl. eis tên polin, in mittelgriech. Aussprache /(i)stimbóli(n)/, > Istambul, Stambul) oder aus der sehr häufigen Wendung tòn kólpon tês Attaleías /tongòlpondisatalías/ > il golfo di Satalía. So haben etwa die Itinerare des Daniel und des Sæwulf (frühes 12. Jh.) Satalia, Eudes de Deuil (Mitte 12. Jh.) 64, 67 usw. Satellia, Wilhelm von Tyrus (spätes 12. Jh.) $16.26 \mathrm{zu}$ a. 1146 Satalia (so nennen es nostri), gulphus Sataliae, der Trojaroman 12329 Satelee, Ambroise 1318 al gofre de Sartalee, Florence de Rome 5590 Satel[l]lie und noch Ariosts Orlando Furioso 17.65.7, 19.46.5 Satalía.

29 Tübinger Atlas des Vorderen Orients, Wiesbaden 1977-1991, Karte B VI 8, Kleinasien: Das Byzantinische Reich (7.-9. Jh.); Islâm Ansiklopedisi, Bd. I, Istambul 1940, s. v. Antalya. Im Abendland wurde die Stadt besonders bekannt, als 1148 Ludwig VII. sich nur mit Mühe vor türkischen Verfolgern in die byzantinische Festung retten konnte.

30 Ich verzichte allerdings darauf, die dunkle Stelle v. $1502 \mathrm{f}$. parallel auszuwerten. Dort kann man Val Metas nicht gut mit Prosper Boissonnade, Du Nouveau sur la Chanson de Roland, Paris 1923, S. 195, als ,Mekka‘ nehmen, da arab. Máqqa, gesprochen etwa /mǽq:æ/, erwartungsgemäß im Frz. vom 12. Jh. bis heute ohne Akzentschwankungen (la) Mecque (im Altfrz. mit den zu erwartenden Varianten) ergibt. Den Galaf<r>es nennt nur O amiralz, CV7T vielmehr 
Von zentraler Bedeutung, wenn auch in der Forschung bisher als Bizarrerie angesehen, scheint mir die Tatsache, dass Marsilies Bruder Falsaron Herr der tere Dat[ha]n e [Abir]un ist (v. 1215). ${ }^{31}$ Dathan und Abiram sind nur aus dem Alten Testament (Numeri 16) bekannt, israelitische Sippen, die nie Land besaßen, sondern auf der Wüstenwanderung im Süden Palästinas infolge ihres Ungehorsams untergingen. Das ,Land von Dathan und Abiram“ kann also nur das Land ihres einstigen Untergangs meinen. Besitzt nun von zwei Brüdern der eine Spanien, der andere eine Herrschaft in Palästina, so heißt das doch wohl, dass beider Vater, aus Palästina stammend, an der Eroberung Spaniens entscheidend beteiligt war und dass das Verhältnis der Brüder trotz der geographischen Entfernung noch ein enges ist. ${ }^{32}$

In v. 1370 fällt ein Justin de Val Ferree, also ,Justin vom mit Eisen versehenen' - d. h. doch wohl: mit Eisen versperrten - ,Tal'. Bisher unbemerkt, taucht er als einziger Komparse des Rolandsliedes im Roman d'Alexandre ${ }^{33}$ wieder auf. Und dort scheint er besser hinzupassen; denn Alexander hat ja nach der Legende eine Gruppe von Barbarenvölkern in der Ebene an Don und Asowschem Meer durch eine porta ferrata an ihrer gebirgigen Grenze von der zivilisierten Welt des Orients abgeschlossen, bis ein König der Hyrkaner - ein Zeitgenosse Vespasians und Titus'- sie wieder freiließ. ${ }^{34}$ Also wieder ein orientalischer Gast bei Marsilie?

In v. 1556 macht Olivier sieben Arrabiz kampfunfähig. Da das Wort später im Lied (v. 3011, 3081, 3481, 3511, 3518, 3640) immer Orientalen bezeichnet, so sind diese mit einer gewissen Wahrscheinlichkeit hier ebenfalls, also als weitere orientalische Freiwillige, gemeint. ${ }^{35}$

amanzor, $\mathrm{P}$ weicht aus auf son seignor, nKV4L scheiden aus. Zu verstehen ist die Stelle wohl so, dass Abisme einst durch einen Teufel einen Helm erhielt, den ihm Galafre - wohl auf dem Totenbett, wo die Teufel schon warteten - vermacht hatte; Galafre wäre demnach zur Zeit der Roncevaux-Schlacht schon tot und konkurriert nicht mit Baligant.

31 So zu Recht alle Editoren. Die Vulgata hat wohlgemerkt Abiron statt des hebr. (und damit heutigen) Abiram. Richtig also nK Datan et Abiron, fast richtig CV7 Datan et d'Abiron. In 0 datliun \& balbiun ist das $h$ von Dathan als li und $\delta(')$ von d'Abiron als $b$ verlesen; vorher hatte sich der Name Albion eingemischt.

32 Ein Korollar aus dieser Tatsache: Der Dichter stellt sich die islamische Eroberung Spaniens, historisch fast richtig, als in der Generation vor Karl geschehen vor. Dass er trotzdem in Spanien schon gar keine Christen mehr kennt, zeigt, dass er den Islam für intoleranter hielt, als dieser war - damals eine französische, nicht spanische Haltung, eine Projektion.

33 The medieval French Roman d'Alexandre, ed. Edward C. Armstrong [et al.], Bd. 2, version d'Alexandre de Paris, Princeton, Princeton U.P., 1937, Ndr. 1965, Branche 3, v. 1725.

34 So z. B. Hegesippus, Historiae [die lat. Fassung des Josephus], ed. Vincenzo Ussani, 2 Bde., Wien 1932, lib. 5, cap. 50, hier Bd. 1, S. 405.

35 So kommentarlos auch Segre [wie Anm. 1] im Namenregister: ,Araber'. Diese Hauptbedeutung von afrz. Ar(r)abi(t) geht einfach zurück auf arab.'arabī ,arabisch` (Volk oder Sprachge- 
Zwischen Orient und Okzident angesiedelt ist Marsilies Vertrauter ${ }^{36}$ Valdabrun, Herr über vierhundert Schiffe und fähig, mit ihrer Hilfe Jerusalem den Christen durch Verrat zu nehmen (v. 1562-1568).

In v. 1613-1652 finden wir unter Marsilies Kriegern auch Grandónies, / Filz Capüel, le rei de Capadoce. Kappadozien kennt der Dichter zumindest aus dem Neuen Testament (Act. Apost. 2.9, 1 Petr. 1.1), kann sich also über seine ungefähre Lage nicht irren. Damit ist Grandonie ein weiterer orientalischer Gast an Marsilies Hof, ein Prinz, der außerhalb des väterlichen Schutzes an einem befreundeten Hof Kriegserfahrung und Kampfesruhm gewinnen will.

Im Pseudo-Turpin kann Roland kurz vor seinem eigenen Tod seinen Verderber Marsilie töten; das ist einfache Epenlogik, ausgleichende Gerechtigkeit, wie wir sie erwarten. Im Rolandslied kann Roland Marsilie nur die rechte Hand abschlagen (v. 1903), ohne im Diesseits zu erfahren, ob Marsilie an dieser Wunde stirbt. Warum? Marsilie verliert damit nicht etwa eine meineidige Schwurhand, denn er hat Karl keinen Eid geleistet und Ganelon seinen Eid gehalten. Der Dichter wird uns zwar leitmotivisch an die Agonie des verblutendeinarmigen Marsilie erinnern (v. 2574, 2701, 2719, 2781, 2795), aber seine eigentliche Begründung findet das Motiv in den beiden großen Szenen, die es ermöglicht. Da ist erstens (v. $2677 \mathrm{f}$.) in schauerlicher epischer Ironie der noch ahnungslose Baligant, der nach seiner Landung in Spanien Marsilie einen goldbesetzten Handschuh übersendet, den dieser über die rechte Hand streifen soll, um sich vor Baligant als dessen Lehnsmann zu bekennen. Und da ist später (v. 2827 ff.) der von zwei Helfern aufgerichtete Marsilie, der den Handschuh mit der linken Hand greifen muss, nicht um sein Lehen anzuerkennen, sondern um es zurückzugeben. Gerade hierdurch wird zwischen den beiden sarazenischen Hauptpersonen eine Verbindung sinnfällig gemacht, die dem Baligant-Teil sehr zugute kommt. Denn indem Baligant ausdrücklich diesen Handschuh annimmt

meinschaft); vgl. auch unten Anm. 39. Dass sie hier vorliegt, ist freilich nicht sicher. Denn der Pseudo-Turpin unterscheidet klar Arabs ,Araber, arabisch“ in der Wendung „regem Arabum“ (cap. 9) gegenüber „milites fortissimi qui vulgo dicuntur Arabit“ (cap. 3) ,in Verbänden zusammenlebende Glaubenskrieger, die militärische und religiöse Exerzitien betreiben'; letzteres gehört laut FEW, Bd. 19, s. v. arab (wo die Vermischung beider Wörter im Altfrz. als möglich bezeichnet wird), zu arab. ar-rābita ,Verband [solcher Krieger]' (vgl. auch arbita, einen der Plurale von ribāt, ,Garnisonen [solcher Krieger]'), von derselben Wurzel wie das an sich gleichbedeutende, sekundär zur Dynastiebezeichnung gewordene al-murābitūn ,Almoraviden`.

36 In O v. 1563 kann man zweifeln, ob Valdabrun (celoi) oder Marsiliun Subjekt ist, O v. 618 spricht trotz Verderbnis für ersteres, was an beiden Stellen durch den $\beta$-Zweig bestätigt wird (der am besten in v. 618 durch CV7, in v. 1563 durch TL repräsentiert ist), vgl. die Edition Segre (wie Anm. 1) ad loc. Dann ist Valdabron der Ältere der beiden, und seine Stellung zwischen Ost und West ist offenbar alt; vgl. das oben zu Falsaron Gesagte. 
(v. 2838), übernimmt er auf seine Person die Bilanz aus Marsilies Situation, d. h., nicht nur die Pflicht, ihn an Karl zu rächen, sondern auch die Verantwortung für seine Taten. So ist es zu verstehen, dass Karl sich - nach der Rache an jenen anonymen Flüchtigen am verlängerten Tag - im Gebet (v. 3109) und in zwei anfeuernden Reden an seine Leute (v. 3411 f., 3627-3629) für Rolands Tod auch an Baligant ,rächen' will. Wenn somit für den Dichter auch die BaligantSchlacht noch Rache für Roland ist, sozusagen eine zweite, überhöhte Rache, so rücken damit beide Teile des Liedes wesentlich näher aneinander, als es zunächst den Anschein hatte. Marsilies abgeschlagene Hand setzt also den Baligant-Teil voraus. (Interpretiert man mit Graevell, Jenkins, Lerch und Steinmeyer $^{37}$ Karls zweiten Traum als Vorschau auf Roncevaux statt auf Ganelons Prozess, so bezöge sich sogar schon v. 732 „La destre oreille al premer ver trenchat“ voraus auf den Verlust von Marsilies rechter Hand und damit indirekt auf den Baligant-Teil. ${ }^{38}$ )

Der Sarazene, der dem bewusstlosen Roland sein Schwert stehlen will (v. 2274-2282), frohlockt: „Iceste espee porterai en Arabe.“ Da er aus Spanien oder (wahrscheinlicher) Nordafrika, nicht aus Arabien stammt, heißt dieser Satz: Er will seine Trophäe nicht nur seinem unmittelbaren Lehnsherrn präsentieren, sondern möchte sie quer durch die Länder des Islams bis in den Orient tragen, zum Lehnsherrn seines Lehnsherrn. ${ }^{39}$

Warum wird Karls Schwert Joiuse gerade in v. 2501-2508 vorgestellt? Im Roncevaux-Teil hat Karl es nicht benötigt; auch die Verfolgung der Flüchtigen während des verlängerten Tages ist mehr das Niedermähen einer anonymen gent criminel als eine Schlacht, an der physisch teilzunehmen des Kaisers Ruhm vermehren könnte. Im Baligant-Teil hingegen wird l'espee de France (v. 3615) das Duell aller Duelle entscheiden. Doch statt es erst im letzten Augenblick ein-

37 Vgl. die Edition Segre (wie Anm. 1) ad. loc.

38 Ich selbst neige allerdings dazu, Karls zweiten Traum auf Ganelons Prozess zu beziehen. Zur Form der Träume sei hier en passant festgestellt, dass entgegen den konsequenten Tierallegorien des zweiten bis vierten Traums der erste Traum (v. 719-723) Ganelon in menschlicher Gestalt zeigt, damit Karl schon von v. 743 an (Ganelon schlägt Roland als Führer der Nachhut vor) einer Erkenntnis von Ganelons Verrat näher kommen kann (vgl. v. 745-747, 771-773, 784786, 823-825, 830-840) als selbst Naimes (v. 774-781, 832). Karls Tragik soll eben darin liegen, dass er entgegen der himmlischen Warnung aus menschlicher Rücksicht auf seinen Schwager vor dem Hof nicht auszusprechen wagt, was er fürchtet, und deshalb auch nicht die Kraft findet, seinem Neffen gegen dessen Willen das halbe Heer als Nachhut aufzudrängen (v. 784791) - auch das ein psychologisch abgründiger Zug: Karl scheitert daran, dass er nicht an die Tiefe menschlicher Bosheit glauben will.

39 Arabe ,Arabien` ist globale Bezeichnung der Weltgegend, aus der Baligant kommt ( islamischer Orient), zumindest noch v. 2810, 2980, 3331, 3555, wahrscheinlich auch 185, 652 (zu beiden s. den Haupttext weiter oben), 3943, enger höchstens 3473. 
zuführen - was auf die Rezipienten wie ein ad-hoc-Einfall wirken könnte -, nützt der Dichter dafür den letzten vorherigen Ruhepunkt der Handlung, nämlich das stimmungsvolle Bild des übermüdeten, in seiner Rüstung schlafenden Kaisers. ${ }^{40}$ Ohne das Duell im Baligant-Teil gäbe es also auch die Schwertbeschreibung nicht.

Mehr noch. Zentrum dieser Beschreibung ist die Christusreliquie. Blicken wir nun zurück auf Rolands Durendal, so finden wir dort zwar eine wohlüberlegte Aufzählung von Reliquien (v. 2345-2348): Basilius vertritt die frühe (noch keiner Trennung von Rom verdächtige) Ostkirche; Dionysius wird seit dem 9. Jh. mit dem Areopagiten identifiziert, erleidet aber sein Martyrium, seinen dies natalis, im Westen und steht damit für diesen, speziell für Frankreich; Petrus vertritt Christi Jüngerschaft, Maria sogar seine Familie. Aber der natürliche Höhepunkt der Aufzählung fehlt: eine Christusreliquie. Sie fehlt, weil der Dichter sie Karls Schwert vorbehalten will. Schon in dieser Roncevaux-Szene par excellence, der Apostrophe an Durendal, denkt der Dichter also auch an Joiuse und damit an den Baligant-Teil und wahrt zwischen beiden Schwertern eine Hierarchie.

In v. 2525-2554 weist Karls Traum von dem teils kosmischen, teils tierischen Feindesheer apokalyptischer Größenordnung und von dem ihm selbst aufgezwungenen Zweikampf voraus auf den Baligant-Teil, was nie bezweifelt worden ist. Doch da die Kampfkraft Spaniens und Afrikas bereits erschöpft ist, wird damit den Rezipienten auch zu verstehen gegeben, dass dieses Heer aus dem islamischen Orient kommen muss. Die Verknüpfung dieser Laisse mit den unmittelbar vorhergehenden hat Burger ${ }^{41}$ herausgearbeitet.

Jeder Rolandleser kennt die heidnische Anti-Trinität Apol(l)in, Mahum(m)et, Tervagan $(t)$. Aber zusammen werden die drei erst v. 2580-91 erwähnt, das heißt, auf der Schwelle zum - bzw. nach Delbouilles, Rychners und Duggans ${ }^{42}$ Teilung schon im - Baligant-Teil, dann v. 2696 f., 2711 f., 3267 f., 3490 f. Vorher nennt das Lied (von v. 8 bis v. 2468) an neun Stellen nur einen, an zwei Stellen zwei von ihnen. Sollen wir glauben, erst der Baligant-Dichter habe in einem Geniestreich die drei schon vorgefundenen Götter zur Anti-Trinität vereint? Liegt

40 Das gestattet dann, schon v. 3146 Baligants Preciuse vorzustellen, wobei aber laut v. 25032511 Joiuse wie das damit zusammenhängende Munjoie nach der Erlösungsfreude des Christen benannt ist, Preciuse also, wie von Baligant nicht anders zu erwarten, ein karikaturhaftmissverstehendes Umschlagen ins Materielle zeigt.

41 André Burger, „Remarques sur la composition de l'épisode de Baligant“, in: Mélanges [...] Maurice Delbouille, Gembloux 1964, Bd. II, S. 59-69, hier 60 f.

42 Delbouille (wie Anm. 13), S. 33, Jean Rychner, La Chanson de geste. Essai sur l'art épique des jongleurs, Genf 1955, S. 39, Duggan (wie Anm. 18), S. 74. 
nicht viel eher ein bewusster Kalkül vor, dergestalt, dass erst nahe dem Höhepunkt durch das Zusammentreten der Trias die geistige anti-trinitarische, d. h. antichristliche Einheit der Heidenheit - ganz wie gleichzeitig ihre militärische Solidarität - manifest werden soll? Dann geschieht die Beschränkung im gesamten Roncevaux-Teil schon mit Blick auf den Baligant-Teil!

In Vers 2602f. ruft Bramimonde aus: „Li amiralz i ferat cuardie, / S’il ne cumbat a cele gent hardie.“ Der bestimmte Artikel in ,suggestiver' Funktion zeigt, dass es für Bramimonde nur einen amiralz gibt, und da eben Spanien und Afrika schon ausgeschieden sind, muss er im Orient sitzen. ${ }^{43}$

Namentlich genannt und charakterisiert wird Baligant erst in v. 26142616, ${ }^{44}$ nämlich in dem Augenblick, als sich die ,Kamera“ des Dichters voll auf den Orient öffnet und zugleich eine eindeutige Rückblende vollzieht, ${ }^{45} \mathrm{zu}$ nächst bis ins erste Jahr von Karls Spanienzug, dann konkreter in den Frühling des Jahres, in dessen Herbst soeben die Schlacht von Roncevaux stattgefunden hat. An dieser Stelle war eine sehr explizite Zeitangabe nötig, und der Dichter hat sie herausgearbeitet durch zitathaften Rückbezug auf Verse der Eingangslaisse des ganzen Rolandsliedes (v. 2609-2611 v. 1-2, 4-5). Doch selbst diese klare Zäsur kann man, wie Burger ${ }^{46}$ meines Erachtens überzeugend dargelegt hat, nicht als Indiz für einen Autorwechsel gelten lassen. Denn schon in v. $703 \mathrm{f}$. findet sich eine ähnliche, wenn auch kleinere Zäsur mit zitathaftem Rückbezug auf dieselben Verse der Eingangslaisse, und beide Zäsuren zusammen gliedern das gesamte Lied auffällig gut: v. 1-702 behandeln die Ursache der RoncevauxSchlacht, Ganelons Verrat, v. 703-2608 die Folgen von Ganelons Verrat, nämlich die Roncevaux-Schlacht einschließlich der Flucht des todwunden Marsilie nach Saragossa, der letzte Teil des Liedes von v. 2609 an dann Gottes Urteil über die Heiden und über den Verräter. Zwar setzen solche zitathaften Rückgriffe mit einer an Sicherheit grenzenden Wahrscheinlichkeit Schriftlichkeit voraus; zudem ist zuzugeben, dass die verflossenen ,sieben Jahre‘ in v. $2609 \mathrm{ff}$. mit mehr Inhalt erfüllt werden als in v. $1 \mathrm{ff} .,{ }^{47}$ indem der Dichter eben achtzehn

43 Wobei allerdings nach der antiken und mittelalterlichen Geographie einschließlich derjenigen des Rolandsliedes die Grenze zwischen Afrika und Asien westlich Alexandria verläuft, Ägypten also (letztlich aus kulturellen Gründen) schon zum Orient gehört; der Golf von Suez war eben vor dem Bau des Kanals für eine mediterran orientierte Geographie uninteressant.

$44 \mathrm{Zu}$ ihnen ausführlich Beckmann (wie Anm. 3), S. 540-542.

45 Vgl. Dorothea Kullmann, „Le début de l'épisode de Baligant“, in: L'épopée romane, Actes $d u X V^{e}$ Congrès international Rencesvals (Poitiers, 21-27 août 2000), hrgg. von Gabriel Bianciotto und Claudio Galderisi, Poitiers 2002, Bd. I, S. 577-587, hier $578 \mathrm{ff}$.

46 Burger (wie Anm. 19), S. 48 f.; vgl. auch dens. (wie Anm. 41), S. $68 \mathrm{f}$.

47 Darauf insistiert Kullmann (wie Anm. 45), S. 580 f., und sieht darin ein Argument für Autorentrennung. 
Verse braucht, um Marsilies dringenden, fast drohenden Hilferuf und Baligants Kriegsvorbereitungen anschaulich zu schildern. Dennoch kann man sich schwer vorstellen, der Baligant-Dichter hätte die beiden ersten Markierungen als ein Doppel erkannt, das er zum Tripel steigern konnte.

Bedeuten nun alle diese Stellen zusammen nicht, dass der Dichter uns von Beginn des Rolandsliedes an beharrlich an die Existenz eines weiten muslimischen Orients glaubt erinnern zu müssen und dass er etwa von v. 1900 an also innerhalb des Roncevaux-Teils noch vor Beginn des Algalife-Aktes, fast fünfhundert Verse vor Rolands Tod! - in kürzer werdenden Abständen überhaupt Verse schreibt, die eindeutig die Kenntnis der Baligant-Handlung voraussetzen ${ }^{48}$ Wird man diesem Sachverhalt noch durch die Annahme von ,Interpolationen' gerecht? Wenn wir uns schon darauf kaprizieren wollen, den Dichter, der uns das erhaltene Rolandslied hinterlassen hat, den ,Baligant-Dichter ${ }^{\text {zu }}$ nennen, scheint er dann nicht auch so tief in den Wortlaut des RoncevauxTeiles eingegriffen $\mathrm{zu}$ haben, dass er diesen Text Vers für Vers ,verantwortet aus seiner Hand geben konnte?

Gewiss könnte man fragen, warum er dann nicht in der Einleitungslaisse bei der Vorstellung Marsilies kurz mitteilt, der sei ein Vasall des orientalischen Baligant. Aber die Antwort ist wohl einfach: Für sein in lehnsrechtlich-hierarchischen Vorstellungen befangenes Publikum wäre das eine Heruntertransformation der Gegner und damit auch der gesamten Roncevaux-Handlung selbst; zum Schaden des alten Hauptteils wäre von Anfang an klar, dass Roland und seine Mitstreiter nie mit dem Herrn der Heidenheit und seinem Hauptheer auch nur in Berührung kommen, ihr Leben vielmehr hauptsächlich im Kampf mit Vasallen von Vasallen opfern.

Noch eines bleibt hier zu bedenken. Die Afrikaner ${ }^{49}$ kämpfen zwar zusammen mit den Spaniern in der Roncevaux-, nicht in der Baligant-Schlacht, doch erscheinen sie zunächst nur marginal (wohl schon v. 812, jedenfalls $1235 \mathrm{f}$. und 1593 f.). Erst als Marsilie mit den Spaniern flieht (v. 1910-1912), treten die Afrikaner in den Fokus, und ihnen gehört zwangsläufig von Oliviers Tod an der übrige Teil der Roncevaux-Schlacht. Auch hier erweitert sich also der geographische Horizont de facto plötzlich von Spanien auf Afrika. Ist es dann überraschend, dass er sich noch einmal, jetzt von Afrika auf den Orient, erweitert? Geschieht die geographische Ausweitung dann nicht in einem mächtigen Dreischritt (zu-

48 Die Forschung ist sich zwar bei der Frage, wo der Beginn des Baligant-Teiles anzusetzen ist, ziemlich uneins, setzt ihn aber frühestens in v. 2476 an (also gerade am Goldenen Schnitt des Rolandsliedes, siehe oben Haupttext bei Anm. 17); vgl. den Forschungsüberblick bei Kullmann (wie Anm. 45), S. 578 Anm. 9.

49 Im Sinne von Anm. 43. 
nächst Spanien - ab v. 1913 offen Afrika - ab v. 2614 offen der Orient), der sogar der Geschichte abgeschaut ist (zunächst nur Spanien - seit 1086/1087 Afrika seit 1097 der Orient)? Und bietet nicht auch der Pseudo-Turpin Ähnliches, wenn dort die Franken nacheinander gegen Spanier (cap. 2 ff.), den Nordafrikaner Aigoland (cap. 6 ff.), den Syrer Ferracutus (cap. 17 ff.) und schließlich die Perser Babylonier Marsirius und Beliguandus (cap. $21 \mathrm{ff}$.) kämpfen? Schließt aus dieser ebenfalls wohlkalkulierten Steigerung irgend jemand gegen die Autoreinheit?

Als Rolands Hornruf im Hauptheer vernommen wird, sucht Ganelon sowohl im Rolandslied als auch im Pseudo-Turpin durch Bagatellisierung dieses Signals Karl von der sofortigen Umkehr abzuhalten. Im Pseudo-Turpin gelingt ihm das für kurze Zeit, dann erfährt Karl durch Turpins Vision und Thierrys Kommen von Rolands Tod, eilt nach Roncevaux, wo er die Gefallenen beklagt, und weiter zu der durch das Sonnenwunder ermöglichten Verfolgung der Feinde. Doch kaum hat er so das Unaufschiebbare getan, lässt er noch in Roncevaux Ganelon den Prozess machen; denn ,viele‘ bezichtigen diesen des Verrats. Ohne dass man von einem Wort erfährt, das Ganelon zu seiner Verteidigung hätte sagen können, wird er, durch das summarisch geschilderte Duell zwischen Pinabel und Thierry des Verrats überführt, von vier Pferden zerrissen. Diese Eile der Verurteilung ist das $\mathrm{zu}$ allen Epochen bis in die jüngste Vergangenheit ,Normale‘: Mit dem während eines Feldzugs gefassten Verräter macht man ,kurzen Prozess'; er wird - wie es ja noch bis zum Ende des Zweiten Weltkriegs üblich war - ,standrechtlich' hingerichtet. ${ }^{50}$ Wieder hält es also der Pseudo-Turpin mit dem ,gesunden Menschenverstand', fast möchte man, ein böses Wort hervorholend, sagen: mit dem ,gesunden Volksempfinden‘. Selbst wenn diese Lösung nicht die chronologische Priorität vor dem Rolandslied für sich haben sollte, drängte sie sich doch so auf, dass sie jedem, der in eigener Verantwortung die Episode erzählen wollte, als Möglichkeit in den Sinn gekommen sein muss.

Doch es ist eine poetisch karge und juristisch nicht unbedenkliche Lösung: Ein improvisiertes, vielleicht nur mäßig qualifiziertes Gericht urteilt unter dem momentanen Druck der ,vielen', und der Verklagte tritt schnell und stumm ab,

50 Freilich wurden in karolingischer Zeit Hochverratsprozesse manchmal mit großem Aufwand auf Reichstagen durchgeführt (so gegen Thassilo 788 in Ingelheim, gegen Bera 820 in Aachen). Dabei ging es zwar nicht um jüngst ,auf frischer Tat‘ in Feldzügen gefasste Personen. Doch will ich keineswegs ausschließen, dass der Rolanddichter von solchen Prozessen direkt (Reichsannalen?) oder indirekt Kenntnis hatte; jedenfalls trifft er das Ambiente solcher Prozesse recht gut. 
ohne die Mittel zu seiner Verteidigung ausschöpfen zu können. Hat nicht gerade der große Verbrecher im juristischen und erst recht im poetischen Sinne Anrecht auf einen großen Prozess? Und muss dieser nicht auch das Anliegen eines großen Königs sein?

Doch ein solcher Prozess lässt sich nicht im Felde improvisieren, er will in der Heimat gründlich vorbereitet sein. Somit besteht hier der wesentliche Unterschied des Rolandsliedes zum Pseudo-Turpin darin, dass Karl den Prozess auf einen (wohl mittwinterlichen, v. 3746) Hoftag in Aachen verschiebt.

Seinem Publikum macht der Dichter diese Verschiebung wieder durch kleine Kunstgriffe plausibel. Nicht die ,vielen' durchschauen Ganelons Verrat, sondern der Klügste, Herzog Naimes. Und da er der Klügste ist, durchschaut er ihn nicht allmählich, sondern gleich nach Rolands doppeltem Hornruf gerade dadurch, dass Ganelon diesen bagatellisiert (v. 1792). Wieder, sozusagen en passant, ein psychologisch bewundernswerter Einfall: Der Verräter decouvriert sich in seinem letzten Schritt, einem Zuviel an List, selbst. Das reicht, nicht zum ,kurzen Prozess' an Ganelon, wohl aber, um ihn in Ketten zu legen und damit zunächst für die Dauer des Feldzugs unschädlich zu machen. Die Misshandlungen des Verdächtigen durch Schergen vor seiner Verurteilung (v. 1821-1829, 3735-3741) missfallen uns heute, waren aber wohl gängige Praxis fast aller vormodernen Rechtsprechung. ${ }^{51}$

Durch die Verschiebung gewinnt der Dichter wieder zweierlei.

Erstens kann er den Prozess eben als große Staatsaktion gestalten. ${ }^{52}$ Die Richter wählt Karl nicht einfach aus Überlebenden von Roncevaux, sondern

51 Wenn Brault (wie Anm. 4), S. 319, hier annimmt, Ganelons Misshandlung sei typologisch der Misshandlung Jesu nachgebildet, wobei „Ganelon at the stake apes Jesus and poses as innocence persecuted, an attitude designed to arouse sympathy on the part of the unwary“, so scheint mir das eine Über-Interpretation schon deshalb, weil Ganelon hier zwangsläufig völlig passiv ist, also nichts zu seiner ,Pose‘ beitragen kann. Gewiss ,äfft‘ der Teufel manchmal Gott nach, und auch im Rolandslied gibt es ,Gegenbildungen“ wie die Verehrung der Antitrinität oder Baligants Benennung seines Schwertes als Preciuse; aber da handelt es sich um Aktivitäten der Gegenseite, und man muss sich fragen, ob nicht die Ausdehnung einer e contrario argumentierenden Typologie auf passive Situationen zum methodischen Irrweg wird.

52 Die Erklärung mancher Details des Prozesses in der Forschung schwankt bis auf den heutigen Tag; vgl. die beiden rezenten Darstellungen: Bob Duivestijn, „,De Guenelun car me jugez le dreit'. Het proces Guenelun in rechtshistorisch perspectief“, in: Karolus rex: studies over de middeleeuwse verhaaltraditie rond Karel de Grote, ed. Bart Besamusca und Jaap Tigelaar, Hilversum 2005, S. 25-36, und Emmanuel Mickel: „The Implications of the Trial of Ganelon“, in: Approaches to teaching the Song of Roland, ed. William W. Kibler, New York 2006, S. 220-231. Demgegenüber hier, à titre de mémoire, das Wichtigste über die Struktur des Prozesses, wie es sich aus den Standardlehrbüchern der Rechtsgeschichte belegen lässt: Vorsitzender des Gerichts ist der König; doch ist er damit (im germanischen und weithin) im mittelalterlichen Recht nur Garant des korrekten Prozessablaufs und Verkünder des Urteils der Richter, nicht 
beruft sie in aller Form aus dem ganzen Reich, von Sachsen bis zur Bretagne (v. 3699-3703). Er will auch hier vorbildlich handeln; nicht seine Macht als König, sondern das Recht selbst soll siegen. Doch dann kommt der Geniestreich des Dichters: Gerade durch diesen noblen Willen droht der König zu scheitern. Denn das ,erregende Moment' des Prozesses, von doppelter psychologischer Illusionslosigkeit, besteht in der Feigheit der Richter (v. 3797-3806), einer Feigheit, die sie, sobald die Gefahr vorüber ist, durch beflissene Strenge (v. 3951, 3960 ff.) zu kompensieren trachten.

selbst Richter. Das Richterkollegium besteht vielmehr grundsätzlich aus ,Pairs‘ des Beklagten (hier im geographisch weitesten Sinne, um dem Prozess Glanz zu geben); deshalb Karls Aufforderung an sie v. 3750 f. sowie später Karls Hilflosigkeit v. 3814-3818 („Vos estes mi felun!“). Prozessgegner sind der König als Lehnherr des getöteten Roland (und, erst durch Thierry konsequent in den Fokus gerückt, aller anderen Roncevaux-Opfer) sowie Ganelon; diese beiden sprechen also die Anklage- (v. 3752-3756) und die Verteidigungsrede (v. 3757-3760, 3768-3778). Die Doppelfunktion des Königs gilt nicht als bedenklich, da sein Gerichtsvorsitz essentiell ein formaler ist. Das Urteil, das die Richter dem Vorsitzenden zur Verkündung vorschlagen (v. 3808-3813), kann nur durch Anbieten des Zweikampfes - seitens einer Partei oder eines im Urteil überstimmten Richters (v. 3806) - angefochten werden (v. 3824-3836). Gegen den Zweikampf als Beweismittel erhoben sich vereinzelte kirchliche Stimmen schon im neunten Jahrhundert, doch noch im Decretum Gratiani (um 1140) nimmt die Kirche zu Gottesurteilen eine ambivalente Haltung ein, erst auf dem Vierten Laterankonzil werden sie 1215 generell verboten, bestehen aber in der Rechtspraxis noch längere Zeit fort (siehe Lexikon des Mittelalters, wie Anm. 17, s. vv. Gottesurteil und Zweikampf; weitere Literatur ebendort). Es bedarf also keiner Rechtfertigung, dass sowohl der Pseudo-Turpin als auch unser Dichter an den Zweikampf als Gottesurteil glauben. Ein König muss sich bei Zweikämpfen aus naheliegenden Gründen prinzipiell vertreten lassen, und wenn Ganelon nicht wesentlich jünger als seine Frau ist, gehört er in die Generation vor Roland, so dass schon aus Altersgründen seine Vertretung durch einen Clangenossen keiner Erklärung bedarf - sofern nicht überhaupt, wie es scheint, zur Zeit der Entstehung beider Texte Ersatzkämpfer schon in weitestem Umfang akzeptiert wurden. Während alle diese Punkte durchaus zur mittelalterlichen Rechtsprechung passen, gilt das im Rolandslied nicht von den Bürgen (pleges, v. 3846). Denn wenn der Dichter hier die alte Einrichtung der Eideshelfer hochtransformiert zu einem Clan von Bürgen, deren Leben am Ausgang des Zweikampfes hängt, so gab es dergleichen nie (Bédier, wie Anm. 13, S. 319) und kann es nie gegeben haben; andernfalls hätten verfeindete Adelsparteien einander in genau diese Position manövriert, ,legal‘ auszulöschen versucht und damit schnellstens das Reich in den Bürgerkrieg gestürzt. Was schließlich die Vierteilung durch Pferde angeht: Nach Verhängung eines Todesurteils erfolgt im mittelalterlichen Gerichtswesen die anschließende Entscheidung über die Hinrichtungsart - in auffälligem Gegensatz zum vorhergehenden Formalismus des Prozesses - unmethodisch-situationsgebunden, je nachdem, wie stark sich die Empörung noch in Strafphantasien austoben muss (vgl. auch den folkloristischen Befund bei Stith Thompson, A Motif-Index of Folk-Literature, 6. Druck, Bloomington 1997, Bd. 5, Q 416: ,Punishment: drawing asunder by horses'); insofern ist die Vierteilung hier die härteste denkbare, aber nicht eigentlich eine ,unhistorische“ Strafe, auch wenn sie später auf Königsmörder beschränkt erscheint (zu letzterem Punkt vgl. Bédier, wie Anm. 13, S. 319 f.). 
Der zweite Gewinn aus der Verschiebung von Ganelons Prozess ist vielleicht der strukturell wichtigere: Durch die Verschiebung stellt der Dichter Ganelons und Bramimondes Schicksale nebeneinander und eröffnet damit einen Blick auf die ganze Breite der condition humaine oder, ohne Anachronismus gesagt, auf zwei Extremfälle von Lebenswegen in die Verdammnis und in das Heil - wobei es dem Lied sehr zugute kommt, dass das helle der beiden Bilder am Schluss steht. Nach überwiegender mittelalterlich-katholischer Theologie gewährt Gott dem Menschen eine zu dessen Seelenrettung ,genügende‘ Gnade (gratia sufficiens), aber nicht jedem von vornherein gleich viel und gleich sichtbare Gnade. Ganelon scheint als Getaufter und mehr noch als hoher Würdenträger in einem christlichen Königreich, ja als Schwager des Beschützers der Christenheit ungewöhnlich nahe seinem Seelenheil aufgestellt; doch er verscherzt sich schrittweise alles und findet nicht einmal am Ende zu einem mea culpa. Bramimonde scheint als Heidin, spezieller als Heidenkönigin und intelligent-aktive Unterstützerin ihres Mannes (v. 634-640) ungewöhnlich weit von ihrem Seelenheil aufgestellt; aber im Leid um ihren Mann wird sie ganz allmählich an ihrem Glauben irre (v. 2576 ff., 2595 ff., 2714 ff., doch vgl. noch einen letzten, fast instinktiven Hilferuf v. 3641) und gelangt eben dadurch zu einer illusionslosen Einschätzung der Realität (v. 2721 f., 2734-2740); deshalb ist sie, statt wie ihr Mann stumpfer Verzweiflung und damit der mors animae $\mathrm{zu}$ verfallen, auch in schwerster Stunde noch zur Übergabe der Festung an Karl fähig (v. 3655 f.). Mit dieser durchaus aktiven Geste überantwortet sie sich auf der literalen Ebene der Gnade des siegreichen Königs, auf der moralischen zeigt sie eine NichtVerzweiflung, die auch beim noch irrenden Menschen gottgefällig ist, und da Karl ja für sie das Christentum verkörpert, dürfen wir hinzufügen: Auf der anagogischen Ebene wird sie bereit, wie halbbewusst auch immer, sich der ihr noch unbekannten Gnade dieses Glaubens zu öffnen. Von nun an kommt ihr diese Gnade auch sichtbar entgegen und gibt Karl eine Ahnung ein von dem moralischen und intellektuellen Potential dieser Frau (v. 3673 f., 3680 f.), das schließlich nach angemessener Vorbereitung (v. 3979) und eigenem Entschluss (v. 3980) in der Taufe (v. $3981 \mathrm{ff}$.) seine wahre Richtung finden wird, symbolisiert durch den Namen einer frühen Christin (v. 3986), die ebenfalls für sich, aber nicht für ihren hochgestellten Ehemann das Heil erringen konnte. ${ }^{53}$

53 Knapp und richtig schon Bédier (wie Anm. 13) S. 320. Über andere, meines Erachtens weniger wichtige Aspekte der Namenwahl (wie die Existenz des Klosters Santillana del Mar) und der ganzen Konversionsszene vgl. Brault (wie Anm. 4) S. 333-335, 474-476. Ein typologischer Einfluss der biblischen Rahab, wie ihn Marianne Cramer Vos, Aspects of Biblical Typology in La Chanson de Roland, Diss. University of Rochester, 1970, S. 156-159, erkennen will, scheint 
An Bramimondes Taufe schließen sich die vieldiskutierten zwölf Schlussverse des Liedes an (v. 3991-4002), die mit ihren unerwarteten Toponymen Bire und Imphe heute oft als Aufruf ihres Dichters an seine Zeitgenossen zu einem Entsatzkreuzzug gedeutet werden in der Hoffnung, damit den für das ganze Lied so erwünschten terminus ad quem zu finden. ${ }^{54}$ Doch was mich an ihnen frappiert, ist ihre, wie ich glaube, reale Funktion im Lied und ihre dichterische Qualität. Unter diesen Aspekten möchte ich sie hier besprechen, ohne, wie einleitend gesagt, ganz sicher zu sein, dass sie ,dem‘ Dichter ,des‘ Rolandsliedes gehören.

Dazu muss ich in gedrängter Form ${ }^{55}$ einige trivialisierende Deutungsmöglichkeiten ausräumen. Erstens: Dass die Episode innerhalb der RolandsliedÜberlieferung nur in $\mathrm{O}$ erscheint, spricht nicht gegen sie, da die anderen Textzeugen schon vorher ausgeschieden sind. ${ }^{56}$ Das Rolandslied ist eben ein Text, dessen Schlussteil nur in einer Handschrift, glücklicherweise der besten, erhalten ist; um Teile eines solchen Textes für ,unecht' $z u$ erklären, müssten sehr gravierende innere Gründe angeführt werden. Zweitens: Bei v. 4002 „Ci falt la geste que Turoldus declinet“ deutet nicht etwa schon die bloße Stellung am Werkende auf einen ,Schreibervermerk'; denn wenige Jahrzehnte (oder nur Jahre?) später unterzeichnet Wace seine Vie de sainte Marguerite mit den Worten „Ci faut sa [scil. Margaretes] vie, ce dit Wace, / Qui de latin en romans mist /

mir nicht vorzuliegen: Weil die Hure Rahab an einen künftigen gottgewollten Sieg der Feinde glaubt, hilft sie ihnen schon, bevor überhaupt der Kampf um ihre Heimatstadt beginnt, erkauft durch ein heimliches Kennzeichen im Voraus ihr eigenes und ihrer Familie Überleben und muss während der Eroberung in ihrem gekennzeichneten Haus bleiben. Der Unterschied zu Bramimonde - und meines Erachtens zugunsten von Bramimonde - könnte kaum größer sein. 54 Dass diese Verse als Datum des Liedes schon einmal 1085 erweisen sollten (Henri Grégoire, „Imphe, la ville d'Amphion en terre d'Épire“, in Mélanges [...] Ernest Hoepffner, Paris 1949, S. 183-190, speziell S. 183 Anm. 1 mit Nennung der einschlägigen Aufsätze Grégoires seit 1939), ist heute so gut wie vergessen. Was ich auch an der Datierung dieser Verse (wenn auch nicht des ganzen Liedes) auf nach 1149 bei de Mandach (wie Anm. 15), S. 199-203, 280, 296-300, noch unbefriedigend finde, habe ich kürzlich an anderer Stelle dargelegt, siehe Beckmann (wie Anm. 25), S. 201 Anm. 6.

55 In den folgenden Anmerkungen nenne ich aus der umfangreichen und kontroversen Literatur zu den Schlussversen nur die Beiträge, denen ich mich verpflichtet fühle. Doch dürfte meine Argumentation zur Genüge zeigen, weshalb ich dieser oder jener von der meinigen abweichenden Auffassung nicht folge.

56 Schon von v. 3682 an sind V4 und die Reimfassungen nur noch ,sporadisch' (Segre, wie Anm. 1, ad v. 3675) vergleichbar; noch weniger trägt K bei, siehe Segres Apparat. (Zu n siehe unten Anm. 64.) Von v. 3975 an, d.h. schon für Bramimondes Konversion, fehlen alle Vergleichsmöglichkeiten, doch wird niemand die Zugehörigkeit dieser Laisse zum Lied bezweifeln, da sie in v. 3674 und 3680 angekündigt wird. In freilich viel vagerem Sinne kann man auch von der letzten Laisse sagen, dass sie in v. 2921-2925 angekündigt wird. 
Ce que Theodimus escrist“, dann seinen Roman de Brut mit „Ci falt la geste des Bretuns / [...] Puis que Deus incarnation / Prist, pur nostre redemption, / Mil e cent cinquante e cinc anz / Fist mestre Wace cest romanz“. ${ }^{57}$ Da überdies decliner trotz gelegentlicher gegenteiliger Behauptungen nicht ,abschreiben', sondern etwa ,paraphrasieren, (mit eigenen Worten) darlegen' bedeutet, ${ }^{58}$ ist mit Turoldus eindeutig mehr als ein Abschreiber, mindestens also ein ,Bearbeiter", anscheinend der ganzen geste, ${ }^{59}$ gemeint - wobei ,Bearbeiter` der höchstmögli-

57 Die erste der beiden Wace-Stellen wurde in unserem Zusammenhang beigebracht von Ernst Robert Curtius, Europäische Literatur und lateinisches Mittelalter, 2. Aufl., Bern 1954, S. 99, die zweite von Burger (wie Anm. 19), S. 58.

58 Von den relevanten Belegen zu lat. declinare, altokzitanisch declinar, afrz. decliner wurden die (beschränkt hilfreichen) antiken zuerst von Leonardo Olschki, „Ci falt la geste...“, Archivum Romanicum 19 (1935), S. 425-431, beigebracht, die wesentlich aussagekräftigeren romanischen von Herbert K. Stone, „Decliner“, Modern Philology 33 (1936), S. 337-350, hier 345-350. Sie sind übrigens zahlreich genug und semantisch nahe genug, um in v. 4002 auch die ganz andere Übersetzung von declinet als ,verfällt körperlich“ (mit dem im Rolandslied seltenen que ,denn') wenig wahrscheinlich zu machen. Dieses ,verfällt körperlich“ würde zudem die Deutung der vorhergehenden elf Verse kaum beeinflussen. Denn selbst ein Autor, der nach nur elf Versen einer völlig neuen Handlung seinen körperlichen Verfall mitteilt oder fingiert, müsste, um überhaupt diese elf Verse noch bringen zu wollen, dieselben Motive haben, wie sie oben im Folgenden vorgeführt werden.

59 La Geste (bzw. l'ancïene geste v. 3742 und Geste Francor v. 1443, 3262) bezeichnet im sonstigen Sprachgebrauch des Rolandsliedes (v. 1443, 1685, 2095, 3181, 3262, 3742 und wohl auch 788) nicht eine Folge von (Groß-)Taten, sondern einen schriftlichen (v. 1443, 3742) Bericht über solche, den sich der Dichter essentiell gleichzeitig mit den Ereignissen (v. 788, 1443, 3181) entstanden denkt und eben deshalb - implizit, aber eindeutig - als autoritativ ausgibt. Doch ist diese seine Quelle real oder fiktiv? Sie müsste nicht nur Roncevaux (v. 1685, 2095) und Ganelons Prozess (v. 3742), sondern sogar die Baligant-Handlung (v. 3262) erzählen und wäre deshalb nicht ,alt‘ (v. 3742), sondern ein Produkt der Zeit nach 1100. Zudem sind alle ihre angeblichen Einzelaussagen verdächtig. Laut v. 1685 soll sie, in unklarer Verbindung mit cartres und brefs, bezeugen, dass schon vor dem letzten heidnischen Sturmangriff Roland, Olivier und Turpin zusammen viertausend Heiden getötet hatten (hier deckt sie eine der quantitativ unwahrscheinlichsten Aussagen des Liedes, und zwar speziell gegen eine Tradition in der Art des Pseudo-Turpin, die wegen des Tötungsverbotes für Geistliche Turpins Teilnahme an der Schlacht von Roncevaux leugnete); laut v. 2095 soll sie, in unklarer Parallele jetzt zu dem heiligen Ägidius, bezeugen, dass Turpin, bis zum letzten Atemzug kämpfend, noch vierhundert Heiden niedermetzelte (hier in besonders klarer Frontstellung gegen die Pseudo-TurpinTradition); laut v. 3262 soll sie gar die dreißig Scharen von Baligants Heer aufzählen (von denen die Pseudo-Turpin-Tradition bekanntlich nichts weiß); und laut v. 3742 bezeugt sie (wieder gegen die Pseudo-Turpin-Tradition), dass Karl den Prozess gegen Ganelon nicht im Felde, sondern mit eigens aufgebotenen Richtern aus vielen seiner Länder in Aachen veranstaltete. Insgesamt ist ihre Neigung zu großen Zahlen ebenso verdächtig wie die Tatsache, dass sie nur genannt wird, wo der Dichter einer speziellen Absicherung bedarf gegen die von dem PseudoTurpin verkörperte Tradition (möglicher-, aber nicht notwendigerweise sogar gegen den 
che hier zu erwartende Grad ist; denn auch ein Dichter in unserem Sinne konnte nicht wagen, sich als Stofferfinder zu erkennen zu geben. Schließlich drittens: Dass eine (direkte oder indirekte) Vorlage von 0 mit v. 4001 an einem Blattende fragmentarisch geendet hätte und der Abschreiber dies in v. 4002 durch „,i falt la geste“ hätte kommentieren wollen (gleichgültig, was er dann mit „que Turoldus declinet“ genau gemeint hätte), ist sehr unwahrscheinlich nicht nur, weil in den gerade genannten Wace-Parallelen ci falt gerade kein Abbrechen, sondern ein natürliches Ende bezeichnet, sondern vor allem, weil diese Vorlage von O dann zwei volle Epen textlich geschickt verbunden haben müsste zu einer Zeit, als sich sonst noch keinerlei Tendenzen zu Sammelhandschriften erkennen lassen. Scheidet also diese Möglichkeit aus, so ist nach v. 4001 das überraschende Abbrechen einer Erzählung, die sich gerade erst zu entfalten begann, ein gewolltes, literarisch verantwortetes.

Ein abruptes Abbrechen der Handlung als literarischer Kunstgriff ${ }^{60}$ und ein Turold als (mindestens) ,Bearbeiter' der geste sollten mehr als ausreichen, den folgenden Versuch einer funktionalen Erklärung der zwölf Schlussverse zu rechtfertigen. ${ }^{61}$

Sind die Verse wirklich ein Kreuzzugsaufruf, so kann man zwar das Abbrechen vordergründig daraus erklären, dass dieser Kreuzzug noch der Zukunft angehört. Doch enden sie - bei dieser Hypothese unerwarteterweise - durchaus nicht exzitatorisch, sondern mit einem Müdigkeitsruf Karls; so bleibt dann nichts übrig, als in diesen Ruf eine implizit-gegenteilige Aufforderung hineinzulesen: Wo Karl müde wurde, müssen ,wir‘ sein Banner aufnehmen.

Aber mag nun ein Kreuzzugsaufruf vorliegen oder nicht - die Verse beziehen sich im Literalsinn auf Karl und müssen deshalb vor allem auf dieser Ebene, der Erzählebene des ganzen Liedes, eine Funktion haben. Wie darf man sich die vorstellen?

Karls Sieg über Baligant hatte nahezu apokalyptische Ausmaße. ${ }^{62}$ Wenn die Rezipienten aus der bis zum Ende des Liedes nachklingenden Hochstimmung

Pseudo-Turpin selbst). Ich halte sie deshalb für fiktiv; der Dichter weiß, dass in hochkarolingischer Zeit Annalen geführt wurden, und hat vielleicht sogar eine vage Vorstellung davon, dass diese Annalenliteratur seine eigene Zeit direkt und indirekt in so vielfältigen Formen erreicht hat, dass man ihn nicht mit Berufung auf eine dieser Formen der ,Lüge‘ zeihen könnte.

60 Echte Parallelen dazu gibt es unter den älteren chansons de geste nicht. Das Wilhelmslied endet zwar pointiert-gedrängt mit einer Erkennungsszene, ist aber narrativ geschlossen. Das Couronnement de Louis wiederum endet mit einem Vers, der sehr vage auf den Charroi de Nîmes verweist, aber nicht den Eindruck erwecken soll, er selbst sei der Anfang einer neuen Handlung. Der ästhetische Effekt ist in beiden Fällen ein ganz anderer als im Rolandslied.

61 Ich erlaube mir im Folgenden auf Formulierungen zurückzugreifen, die ich in meiner Monographie zur Karlamagnús-Saga I (wie Anm. 25), S. 200 f., benutzt habe.

62 Freilich ist in der Erzähltradition wahrscheinlich das Sonnenwunder, möglicherweise auch die Einnahme Saragossas älter als die Entstehung des Baligant-Teiles. Theoretisch könnte also 
heraustraten, erwartete sie eine ganz andere Wirklichkeit. Selbst Saragossa war nach mehrhundertjähriger unangefochtener muslimischer Herrschaft (bestenfalls) vor einigen Jahren oder Jahrzehnten zurückerobert worden, sonst war in Spanien, Nordafrika, dem Orient der Islam ungebrochen aktiv, und zumindest die Kreuzfahrerstaaten konnten ständigen Nachschub von christlichen Kämpfern gebrauchen. Wo waren nur die Früchte von Karls Sieg geblieben? Bei solchen frontalen Zusammenstößen zwischen Poesie und Wirklichkeit hat es die Poesie $\mathrm{zu}$ allen Zeiten schwer, ihr Terrain zu behaupten; im zwölften Jahrhundert drohte ihr, wie gesagt, die globale Verwerfung als ,unwahr‘.

Ich glaube deshalb, dass den Rezipienten nicht nur, wie oben ausführlich gezeigt, der Eintritt in die Baligant-Dimensionen, sondern jetzt durch die Schlussverse auch die Rückkehr aus den Baligant-Dimensionen in die raue Realität erleichtert werden sollte. Doch ist das offensichtlich nicht zwischen zwei Szenen des Liedes möglich, sondern erst ganz an dessen Ende - und selbst dort nur in gedrängtester Form, wenn die Passage nicht ein die Struktur des Gesamtliedes schädigendes Eigengewicht gewinnen soll.

Deshalb werden die Rezipienten mit der Schlussepisode daran erinnert, dass dem Christen - wie ja in aller Eindringlichkeit Jesu Abschiedsreden (Matth. 24 f. Markus $13 \sim$ Lukas 21) und die Offenbarung Johannis lehren - bis zur Wiederkunft Christi kein irgendwie endgültiger Sieg im Diesseits verheißen ist. Weil der darin liegende fortwährende Anspruch Gottes an seine Christenheit die Spanne des einzelnen menschlichen Lebens transzendiert, können die nicht enden wollenden Mühen dem alternden Menschen das Gefühl der Überforderung eingeben; ein solcher Mensch hat - wie einst Hiob, so jetzt Karl (v. 3999-4001) - sehr wohl ein Recht zur Klage, ist aber dadurch nicht der Pflicht enthoben, das ihm Mögliche zu tun. Aus diesem Grunde soll das Lied noch, wie Brault zu Recht sagt, „on a plaintive note“ durch „the prospect of never-ending warfare“ zeigen, dass „man must struggle uncessingly if he is to gain eternal life." ${ }^{63}$ Doch gerade deshalb soll die Episode wenigstens innerhalb des Rolandsliedes ohne Andeutung eines Endes bleiben. ${ }^{64}$ Denn hier verschöbe die Nachricht von einem Sieg das Problem

auch auf dieser Stufe schon ein Erzähler das Bedürfnis nach abschließender Dämpfung der Siegesstimmung empfunden haben; dann wäre die Hoffnung, in den Versen einen terminus ad quem für das ganze erhaltene Lied zu finden, schon prinzipiell dahin.

63 Brault (wie Anm. 4), S. 337, 477.

64 Zwar ist volsist (v. 3999) an der einzigen anderen Vorkommensstelle (v. 332) ein klarer Irrealis, so dass wir auch bei v. 3999 immerhin vermuten dürfen, dass Karl sich der Aufforderung nicht endgültig entziehen wird. Doch selbst dass in der Karlamagnús-Saga, hier nur repräsentiert durch ihr dänisches Derivat, die Episode nicht als Aufruf, sondern als Erzählung bis zu einem für die Christen siegreichen Ende durchgeführt ist (übrigens mit sehr geringen narrativen Kosten), beweist nicht, dass auch der Rolanddichter sie schon so kannte; denn die Andeutung im Rolandslied musste in den folgenden einhundert Jahren sehr dazu verlocken, sie zu 
nur um eine Stufe, eine Niederlage erschiene wie ein zynischer Schlusspunkt unter die Karlsepik, ein Kompromissfrieden wäre des Kaisers unwürdig, einen Kampfestod schließlich konnte Karl nicht sterben, weil allzu bekannt war, dass er in Aachen eines natürlichen Todes gestorben war. ${ }^{65}$ Auch auf dieser Ebene ergibt sich also als anagogischer Sinn des Abbrechens etwa: ,Handelt so, als ob der hier begonnene Krieg noch andauere - denn er dauert noch an'. Das ist ein Aufruf nicht notwendigerweise zu einem konkreten Kreuzzug, aber doch zu einer kreuzzugsbereiten Grundhaltung.

Nun kann aber ein Erzähler nicht ganz ohne formales Schluss-Signal, ohne ,Versieglung، seiner Geschichte aufhören; das sähe so aus, als hätten er oder seine Kopisten durch irgendeinen Zufall die Feder aus der Hand gelegt. Hier reichte zu einer meisterhaft knappen Versieglung der letzte Vers: Ci falt la geste que Turoldus declinet. Und der hat bemerkenswerterweise etwas gemeinsam mit dem ersten Vers des Liedes, nämlich den latinisierenden Charakter. Verglichen mit der Exordialtopik (weniger der Schlusstopik) antiker und mittellateinischer Autoren mutet das bescheiden an, anders jedoch innerhalb des sonst mit Latinismen sparsamen ${ }^{66}$ Rolandsliedes. Wer immer das Rolandslied - oder auch nur, wenn man unbedingt chorizontisch denken will, wesentliche Teile davon dichtete, war ein Kleriker und lebte damit zuvörderst in einem lateinsprachigen Diskursuniversum; alle oder so gut wie alle Handschriften, die er seit seiner Jugend je in den Kloster- oder Dombibliotheken gesehen hatte, waren lateinisch. Deshalb baute er sich und den gelehrten unter seinen Rezipienten beim Eintritt in das noch seltene Milieu einer verschrifteten Muttersprache eine kleine, ,halblateinische“ Brücke: „Carles li reis, nostre emperere magnes“. Auch ästhetisch ein bewundernswerter Vers; denn hier bringt die Tmesis Carle(s)...magnes die

,komplettieren'. Aber auch wenn der Rolandsdichter die Episode schon so gekannt haben sollte, bleibt entscheidend, dass er sie für seine Rezipienten beschnitt und als bloßen Aufruf stilisierte. Zum Problem vgl. Beckmann (wie Anm. 25), S. 200-204.

65 So bekanntlich die gesamte historiographische Tradition von den Reichsannalen und Einhart an, doch nicht nur sie. Zur Bekanntheit von Karls natürlichem Tod auch in der Epik vgl. jetzt Beckmann (wie Anm. 25), S. 212-219, speziell 218 mit Anm. 49.

66 Ebenso eindeutige Latinismen wie die hier zu behandelnden Turoldus und magnes sind höchstens noch Tere Major v. 600 u. ö. (insgesamt sechsmal - falls es ,Land der Ahnen“ bedeutet), (gent / enseigne) paienur v. 1221, 2639, Sathanas v. 1268, Geste Francor v. 1443, 3262, seintisme v. 2344, Orïente v. 3594 (gegen Orïent v. 401, 558) und omnipotente v. 3599 (wo beidemal -e das lat. -em nachahmt) sowie wohl Veire Paterne v. 2384, 3100 - also ein klarer Latinismus auf durchschnittlich 250 Verse. Mit decliner wären mehr Wörter vergleichbar, in den ersten sechshundert Versen etwa humilitet v. 73, glorïus v. 124, 429, poësteïfs v. 460, enluminét v. 535 , martírie v. 591 und vielleicht chrestientet 431, also schätzungsweise eines auf je achtzig bis hundert Verse. Angesichts dieser Zahlen kann man den latinisierenden Charakter gerade des ersten und des letzten Verses nicht für Zufall halten. 
Rezipienten dazu, das zum bloßen Namensteil herabgesunkene magnes von neuem, aus lateinisch magnus heraus, mit Leuchtkraft zu erfüllen. ${ }^{67}$ Entsprechend führt der letzte Vers über eine kleine, ,halblateinische‘ Brücke zurück: Nicht nur ist decliner hier aus semantischen Gründen ein Latinismus; auch konnte der Dichter, als er aus einem Werk, worin in gut muttersprachlichem Stilgefühl Turpin nie Turpinus genannt wurde, in seine lateinsprachige Bildungswelt zurücktrat, sich ohne Prätention so unterzeichnen, wie er es als Redaktor oder Zeuge einer - damals noch selbstverständlich lateinischen - Urkunde getan hätte: Turoldus. Ist diese Komplementarität des ersten und des letzten Verses wirklich Zufall?

67 Das so wiederbelebte magnes benutzt er noch dreimal (v. 2321, 3611, 3622) nach reis, dazu achtmal (v. 703 u. ö.) im formelhaften Anvers Carles li magnes, aber nie mit anderem Bezug als dem auf Karl. 